\title{
Supplementary Information for $\mathrm{Cu}_{20} \mathrm{Si}_{12}$ : A Hollow Cage Constituted of a Copper Dodecahedron and a Silicon Icosahedron
}

\author{
Hui-Yan Zhao, ${ }^{\dagger, *}$ Jing Wang, ${ }^{\dagger, *}$ Ling-Yan Ai, ${ }^{\dagger, \S}$ and Ying Liu ${ }^{*},+*$ \\ †Department of Physics and Hebei Advanced Thin Film Laboratory, Hebei Normal University, Shijiazhuang 050024, Hebei, China \\ National Key Laboratory for Materials Simulation and Design, Beijing 100083, China

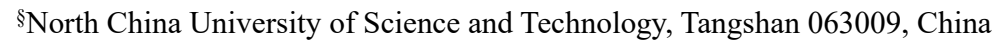 \\ E-mail: yliu@hebtu.edu.cn
}

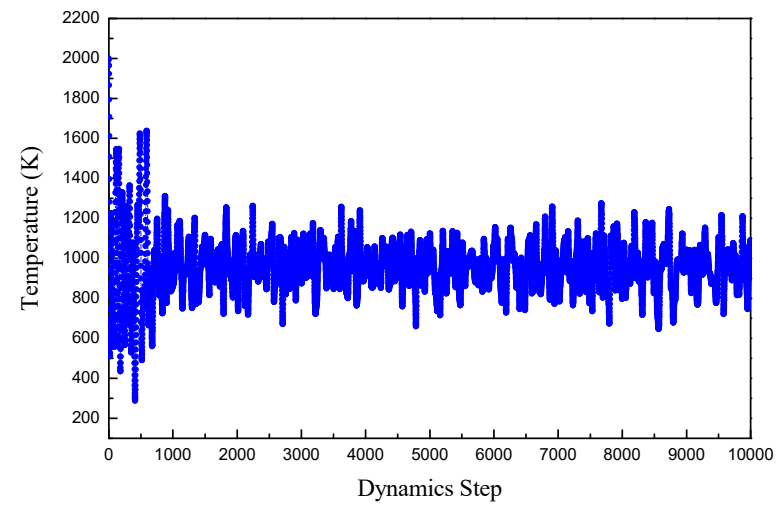

Figure S1. Temperature vs simulation time for the $10 \mathrm{ps}$ MD calculations at initial temperature $T_{\text {ini }}$ of $2000 \mathrm{~K}$.

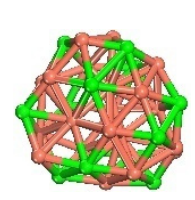

1.211

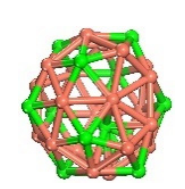

2.492

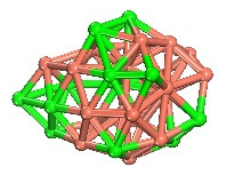

3.635

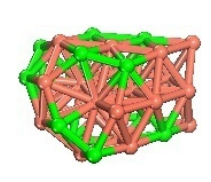

1.899

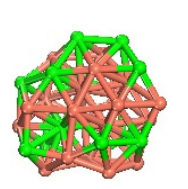

2.681

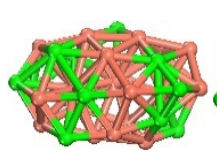

3.706

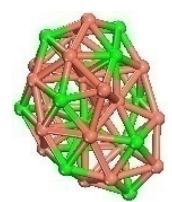

2.011

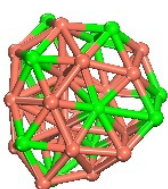

2.801

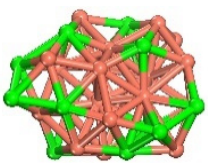

4.047
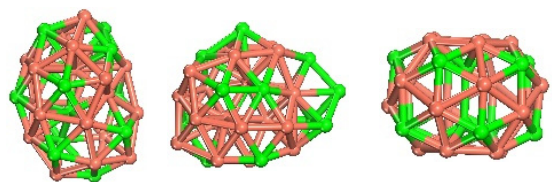

2.145

2.273

2.452
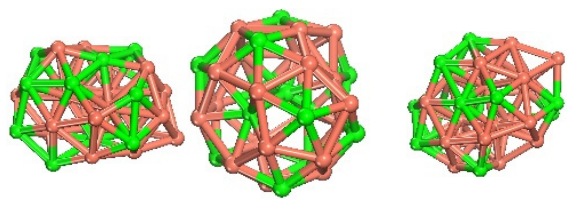

2.906

3.081

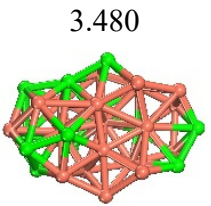

4.539

Figure S2. Optimized isomers based on various structures obtained from the NVE MD calculations at an initial temperature of $3000 \mathrm{~K}$. Below the structures is the binding energy relative to that of the cage-like $\mathrm{Cu}_{20} \mathrm{Si}_{12}$ structure. 


\begin{tabular}{|c|c|c|c|c|}
\hline Isomers & (a) & (b) & (c) & $(d)$ \\
\hline \multicolumn{5}{|l|}{$\begin{array}{c}\text { Initial } \\
\text { structures }\end{array}$} \\
\hline$d_{\mathrm{Cu}-\mathrm{Cu}}$ & 3.746 & 2.929 & 2.575 & 3.746 \\
\hline \multicolumn{5}{|l|}{$\begin{array}{l}\text { Optimized } \\
\text { structures }\end{array}$} \\
\hline$\Delta E_{\mathrm{b}}$ & 0.000 & 0.000 & 0.999 & 2.911 \\
\hline Isomers & (e) & $(f)$ & $(g)$ & (h) \\
\hline \multicolumn{5}{|l|}{$\begin{array}{c}\text { Initial } \\
\text { structures }\end{array}$} \\
\hline$d_{\mathrm{Cu}-\mathrm{Cu}}$ & 4.131 & 4.131 & 2.941 & 6.098 \\
\hline \multicolumn{5}{|l|}{ Optimized } \\
\hline$\Delta E_{\mathrm{b}}$ & 0.002 & 0.001 & 1.500 & 3.577 \\
\hline
\end{tabular}

Figure S3. The initial and optimized structures obtained by distorting the cage-like $\mathrm{Cu}_{20} \mathrm{Si}_{12}$ structure. $d_{\mathrm{Cu}-\mathrm{Cu}}$ represents the distance between $\mathrm{Cu}$ atoms located in the center of the top and bottom sufaces for a flattened initial structure. Here $\Delta E_{\mathrm{b}}(\mathrm{eV})$ is given as the binding energy relative to that of the cage-like $\mathrm{Cu}_{20} \mathrm{Si}_{12}$ structure studied. 


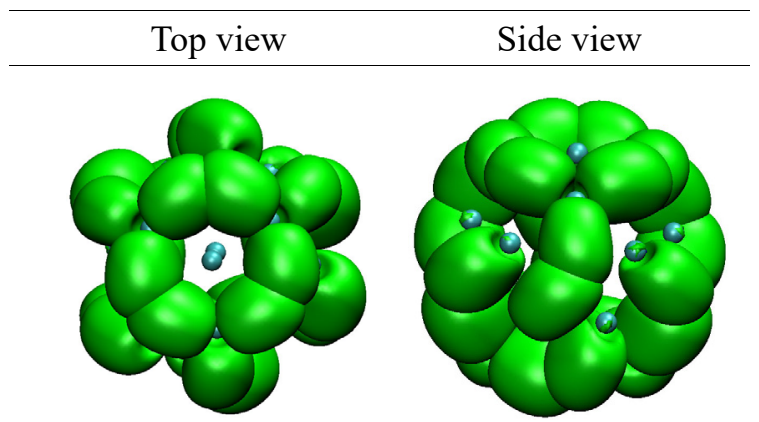

Figure S4. Top view and side view for the superposition of the $\sigma(\mathrm{Cu}-\mathrm{Si})$ bonds in the $\mathrm{Cu}_{20} \mathrm{Si}_{12}$ molecule.

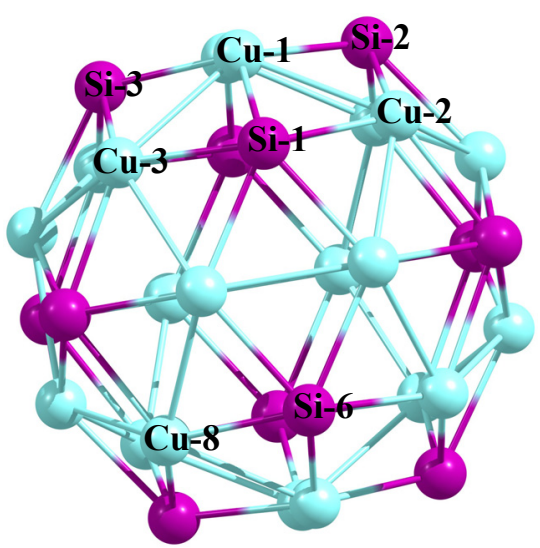

Figure S5. The $\mathrm{Cu}_{20} \mathrm{Si}_{12}$ geometrical structure with showing atom types and the atom numbers in groups of same atom type. 


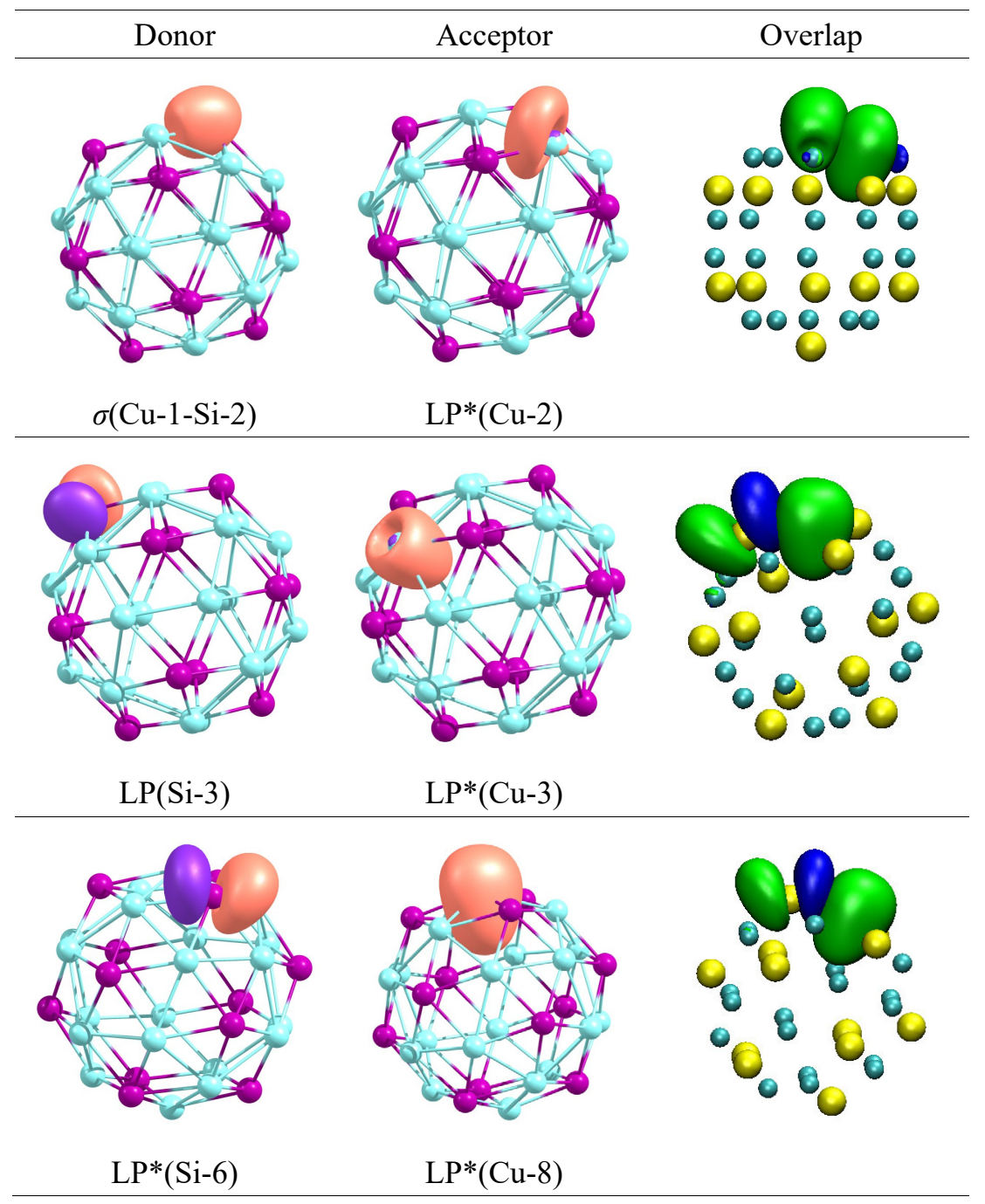

Figure S6. Selected NBO orbitals corresponding to the stronger donor-acceptor interactions, and the overlaps of the donor orbitals and acceptor orbitals.

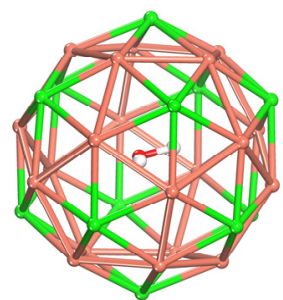

$\mathrm{H}_{2} \mathrm{O} @ \mathrm{Cu}_{20} \mathrm{Si}_{12}$

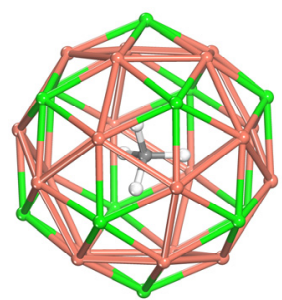

$\mathrm{CH}_{4} @ \mathrm{Cu}_{20} \mathrm{Si}_{12}$

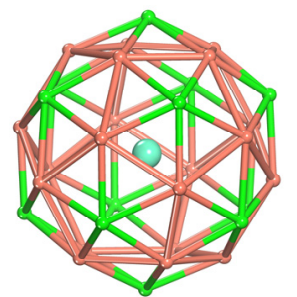

$\mathrm{Eu} @ \mathrm{Cu}_{20} \mathrm{Si}_{12}$

Figure S7. Optimized atom/molecule-encaged $\mathrm{Cu}_{20} \mathrm{Si}_{12}$ structure. Below each structure is the stoichiometry. 


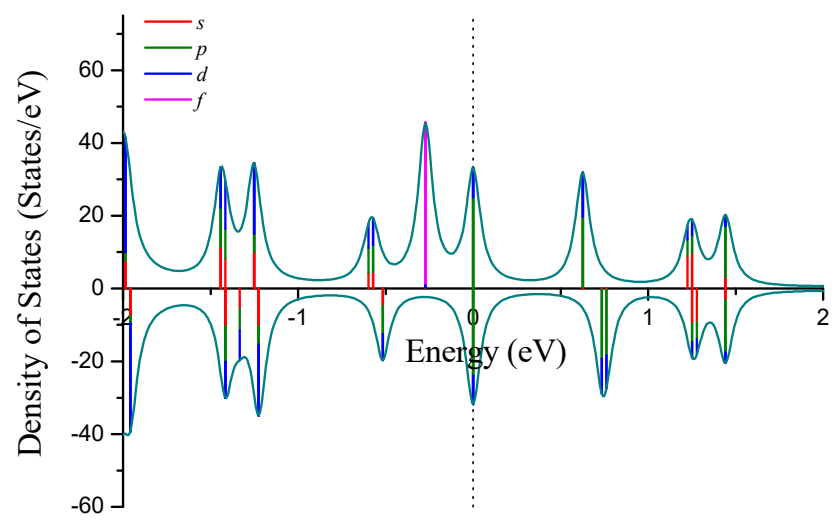

Figure S8 Spin partial density of states for the Eu-encapsulated $\mathrm{Cu}_{20} \mathrm{Si}_{12}$ structure. A Gaussian broadening of 0.02 was used. The Fermi level is shown by the dotted vertical line. Here the vertical lines in the PDOS envelope curve show the relative magnitudes of the electron states in various molecular orbitals. Red line: $s$ electrons; green line: $p$ electrons; blue line: $d$ electrons; magenta line: $f$ electrons.

Table S1 Hirshfeld population analysis for the cage-like $\mathrm{Cu}_{20} \mathrm{Si}_{12}$ structure.

\begin{tabular}{cccccccccc}
\hline Atoms & Number & Charge $(e)$ & Spin $\left(\mu_{\mathrm{B}}\right)$ & & Atoms & Number & Charge $(e)$ & Spin $\left(\mu_{\mathrm{B}}\right)$ \\
\hline $\mathrm{yynnnnnnyyy} \mathrm{Cu}$ & 1 & 0.084 & 0.000 & & $\mathrm{Cu}$ & 17 & 0.084 & 0.000 \\
$\mathrm{Cu}$ & 2 & 0.084 & 0.000 & & $\mathrm{Cu}$ & 18 & 0.084 & 0.000 \\
$\mathrm{Cu}$ & 3 & 0.084 & 0.000 & & $\mathrm{Cu}$ & 19 & 0.084 & 0.000 \\
$\mathrm{Cu}$ & 4 & 0.084 & 0.000 & & $\mathrm{Cu}$ & 20 & 0.084 & 0.000 \\
$\mathrm{Cu}$ & 5 & 0.084 & 0.000 & & $\mathrm{Si}$ & 1 & -0.140 & 0.000 \\
$\mathrm{Cu}$ & 6 & 0.084 & 0.000 & & $\mathrm{Si}$ & 2 & -0.141 & 0.000 \\
$\mathrm{Cu}$ & 7 & 0.085 & 0.000 & & $\mathrm{Si}$ & 3 & -0.140 & 0.000 \\
$\mathrm{Cu}$ & 8 & 0.084 & 0.000 & & $\mathrm{Si}$ & 4 & -0.141 & 0.000 \\
$\mathrm{Cu}$ & 9 & 0.084 & 0.000 & & $\mathrm{Si}$ & 5 & -0.141 & 0.000 \\
$\mathrm{Cu}$ & 10 & 0.084 & 0.000 & & $\mathrm{Si}$ & 6 & -0.140 & 0.000 \\
$\mathrm{Cu}$ & 11 & 0.085 & 0.000 & & $\mathrm{Si}$ & 7 & -0.141 & 0.000 \\
$\mathrm{Cu}$ & 12 & 0.084 & 0.000 & & $\mathrm{Si}$ & 8 & -0.141 & 0.000 \\
$\mathrm{Cu}$ & 13 & 0.084 & 0.000 & & $\mathrm{Si}$ & 9 & -0.140 & 0.000 \\
$\mathrm{Cu}$ & 14 & 0.084 & 0.000 & & $\mathrm{Si}$ & 10 & -0.140 & 0.000 \\
$\mathrm{Cu}$ & 15 & 0.085 & 0.000 & & $\mathrm{Si}$ & 11 & -0.141 & 0.000 \\
$\mathrm{Cu}$ & 16 & 0.084 & 0.000 & & $\mathrm{Si}$ & 12 & -0.140 & 0.000 \\
\hline
\end{tabular}


Table S2 The NBO atomic charges $(e)$ of the $\mathrm{Cu}_{20} \mathrm{Si}_{12}$ cage calculated with the Def2-SV(P) basis set.

\begin{tabular}{|c|c|c|c|c|c|}
\hline Atom & No & $\begin{array}{l}\text { Natural } \\
\text { Charge }\end{array}$ & Atom & No & $\begin{array}{l}\text { Natural } \\
\text { Charge }\end{array}$ \\
\hline $\mathrm{Cu}$ & 1 & 0.057 & $\mathrm{Cu}$ & 17 & 0.062 \\
\hline $\mathrm{Cu}$ & 2 & 0.061 & $\mathrm{Cu}$ & 18 & 0.057 \\
\hline $\mathrm{Cu}$ & 3 & 0.062 & $\mathrm{Cu}$ & 19 & 0.058 \\
\hline $\mathrm{Cu}$ & 4 & 0.055 & $\mathrm{Cu}$ & 20 & 0.058 \\
\hline $\mathrm{Cu}$ & 5 & 0.060 & $\mathrm{Si}$ & 1 & -0.099 \\
\hline $\mathrm{Cu}$ & 6 & 0.061 & $\mathrm{Si}$ & 2 & -0.099 \\
\hline $\mathrm{Cu}$ & 7 & 0.061 & $\mathrm{Si}$ & 3 & -0.098 \\
\hline $\mathrm{Cu}$ & 8 & 0.059 & $\mathrm{Si}$ & 4 & -0.100 \\
\hline $\mathrm{Cu}$ & 9 & 0.058 & $\mathrm{Si}$ & 5 & -0.099 \\
\hline $\mathrm{Cu}$ & 10 & 0.059 & $\mathrm{Si}$ & 6 & -0.097 \\
\hline $\mathrm{Cu}$ & 11 & 0.060 & $\mathrm{Si}$ & 7 & -0.100 \\
\hline $\mathrm{Cu}$ & 12 & 0.058 & $\mathrm{Si}$ & 8 & -0.098 \\
\hline $\mathrm{Cu}$ & 13 & 0.060 & $\mathrm{Si}$ & 9 & -0.097 \\
\hline $\mathrm{Cu}$ & 14 & 0.056 & $\mathrm{Si}$ & 10 & -0.098 \\
\hline $\mathrm{Cu}$ & 15 & 0.061 & $\mathrm{Si}$ & 11 & -0.100 \\
\hline $\mathrm{Cu}$ & 16 & 0.060 & $\mathrm{Si}$ & 12 & -0.100 \\
\hline
\end{tabular}

Table S3 Selected NBO Orbitals of the $\mathrm{Cu}_{20} \mathrm{Si}_{12}$ cage. Their Description and Occupancy (e), Second-Order Interaction Energies $\left(E^{2}, \mathrm{kcal} / \mathrm{mol}\right)$ between Donor and Acceptor Orbitals. Some atom labels are shown in Figure S5.

\begin{tabular}{llll}
\hline NBO orbital $^{a}$ & description of the orbital $\left.^{2.07}\right)_{\mathrm{Cu}}+0.899\left(s p^{1.07}\right) \mathrm{Si}$ & Occupancy & $E^{2}$ \\
\hline$\sigma(\mathrm{Cu}-1-\mathrm{Si}-1)$ & $0.439\left(s p^{2}\right.$ & \\
$\mathrm{LP}(\mathrm{Cu}-1)$ & $d$ & 1.988 & \\
$\mathrm{LP} *(\mathrm{Cu}-1)$ & $p$ & 0.113 & \\
$\mathrm{LP}(\mathrm{Si}-1)$ & $p$ & 0.974 & \\
$\mathrm{LP}^{*}(\mathrm{Si}-1)$ & $p$ & 0.667 & \\
& $\sigma(\mathrm{Cu}-1-\mathrm{Si}-2) \rightarrow \mathrm{LP}(\mathrm{Cu}-2)$ & & 76 \\
& $\mathrm{LP}(\mathrm{Si}-3) \rightarrow \mathrm{LP}(\mathrm{Cu}-3)$ & & 146 \\
& $\mathrm{LP}(\mathrm{Si}-6) \rightarrow \mathrm{LP}(\mathrm{Cu}-8)$ & & 146 \\
\hline
\end{tabular}


Table S4 Component distribution for the orbitals of the cage-like $\mathrm{Cu}_{20} \mathrm{Si}_{12}$ structure. The energy of the HOMO level is set as $0.000 \mathrm{eV}$.

\begin{tabular}{|c|c|c|c|c|c|c|c|c|}
\hline \multirow{2}{*}{\multicolumn{2}{|c|}{ Occupation }} & \multirow{3}{*}{$\begin{array}{l}\text { Orbital } \\
\text { Energy } \\
3 s 0\end{array}$} & \multirow{3}{*}{$\begin{array}{c}\text { HOMO-9 } \\
-1.199 \\
0.005433\end{array}$} & \multirow{3}{*}{$\begin{array}{c}\text { HOMO-8 } \\
-1.192 \\
0.004370\end{array}$} & \multirow{3}{*}{$\begin{array}{c}\text { HOMO-5 } \\
-0.429 \\
-0.00459\end{array}$} & \multirow{3}{*}{$\begin{array}{c}\text { HOMO } \\
0.000 \\
0.000007\end{array}$} & \multirow{3}{*}{$\begin{array}{c}\text { LUMO } \\
1.097 \\
-0.00093\end{array}$} & \multirow{3}{*}{$\begin{array}{c}\text { LUMO+5 } \\
1.324 \\
0.001259\end{array}$} \\
\hline & & & & & & & & \\
\hline $\mathrm{Cu}$ & 1 & & & & & & & \\
\hline $\mathrm{Cu}$ & 1 & $4 s 0$ & -0.13220 & 0.023053 & 0.033694 & -0.00020 & -0.00198 & 0.081568 \\
\hline $\mathrm{Cu}$ & 1 & $4 s 0$ & -0.00154 & -0.00642 & 0.008858 & $-4.4 \mathrm{E}-05$ & 0.005528 & -0.00484 \\
\hline $\mathrm{Cu}$ & 1 & $3 p-1$ & -0.00651 & -0.00575 & 0.003150 & -0.00395 & 0.009841 & 0.005331 \\
\hline $\mathrm{Cu}$ & 1 & $4 p-1$ & 0.024183 & 0.025509 & -0.01618 & 0.006617 & -0.03696 & 0.001085 \\
\hline $\mathrm{Cu}$ & 1 & $3 p 0$ & -0.00032 & -0.00812 & 0.008258 & -0.00871 & -0.00661 & -0.00913 \\
\hline $\mathrm{Cu}$ & 1 & $4 p 0$ & -0.00047 & 0.039254 & -0.03673 & 0.040251 & 0.014199 & 0.040049 \\
\hline $\mathrm{Cu}$ & 1 & $3 p 1$ & 0.006702 & -0.00352 & 0.002907 & & 0.011620 & -0.00018 \\
\hline $\mathrm{Cu}$ & 1 & $4 p 1$ & -0.03178 & 0.016637 & -0.01360 & -0.09946 & -0.03831 & 0.009493 \\
\hline $\mathrm{Cu}$ & 1 & $3 d-2$ & 0.006097 & 0.072241 & -0.02785 & 0.044417 & -0.08640 & -0.11929 \\
\hline $\mathrm{Cu}$ & 1 & $3 d-2$ & -0.00155 & 0.004640 & -0.00573 & 0.006164 & -0.00372 & -0.01026 \\
\hline $\mathrm{Cu}$ & 1 & $3 d-1$ & -0.07248 & 0.059693 & 0.014798 & -0.00731 & -0.01596 & 0.035587 \\
\hline $\mathrm{Cu}$ & 1 & $3 d-1$ & -0.00620 & 0.0060 & 0.002797 & -0.00354 & -0.00 & 0.005928 \\
\hline $\mathrm{Cu}$ & 1 & $3 d 0$ & 0.03 & 0.077013 & -0.12 & 0.039 & 0.102 & -0.02 \\
\hline $\mathrm{Cu}$ & 1 & $3 d 0$ & 0.001031 & 0.006262 & -0.01439 & 0.001349 & 0.015037 & 0.001326 \\
\hline $\mathrm{Cu}$ & 1 & $3 d 1$ & -0.02246 & 0.031417 & -0.00511 & -0.05 & -0.02944 & 0.067799 \\
\hline $\mathrm{Cu}$ & 1 & $3 d 1$ & -0.00 & 0.00 & 0.00 & & -0.0 & 0.008605 \\
\hline $\mathrm{Cu}$ & 1 & $3 d 2$ & -0.12274 & -0.00452 & -0.02608 & -0.00746 & 0.020981 & 0.008389 \\
\hline $\mathrm{Cu}$ & 1 & $3 d 2$ & -0.00590 & -0.00061 & -0.00289 & -0.00258 & 0.000022 & 0.000896 \\
\hline $\mathrm{Cu}$ & 2 & $3 s 0$ & 0.0043 & 0.006 & -0.00779 & 0.000001 & 0.002071 & 0.002940 \\
\hline $\mathrm{Cu}$ & 2 & & -0.10 & & 0.05 & & & \\
\hline $\mathrm{Cu}$ & 2 & $4 s 0$ & -0.00134 & -0.00698 & 0.015014 & $-6.4 \mathrm{E}-05$ & -0.01 & -0.01079 \\
\hline $\mathrm{Cu}$ & 2 & $3 p-1$ & 0.004482 & -0.00913 & 0.012058 & -0.00071 & -0.00874 & -0.00575 \\
\hline $\mathrm{Cu}$ & 2 & $4 p-1$ & -0.02170 & 0.0406 & & & & 0.057761 \\
\hline $\mathrm{Cu}$ & 2 & $3 p 0$ & -0.00041 & -0.00364 & 0.003 & 0.02121 & 0.009372 & -0.00572 \\
\hline $\mathrm{Cu}$ & 2 & $4 p 0$ & -0.00251 & 0.016922 & -0.01362 & -0.07252 & -0.01632 & 0.022774 \\
\hline $\mathrm{Cu}$ & 2 & $3 p 1$ & -0.00813 & -0.00498 & 0.009115 & -0.0059 & -0.00526 & -0.00792 \\
\hline $\mathrm{Cu}$ & 2 & $4 p 1$ & 0.033606 & 0.023089 & -0.04155 & 0.026713 & 0.024380 & 0.049146 \\
\hline $\mathrm{Cu}$ & 2 & $3 d-2$ & 0.065497 & 0.052132 & -0.06593 & -0.03184 & 0.013008 & 0.050379 \\
\hline $\mathrm{Cu}$ & 2 & $3 d-2$ & 0.001235 & 0.005301 & -0.00650 & -0.00150 & 0.001190 & 0.012925 \\
\hline $\mathrm{Cu}$ & 2 & $3 d-1$ & -0.12029 & 0.04028 & -0.02959 & -0.03194 & -0.01080 & 0.028590 \\
\hline $\mathrm{Cu}$ & 2 & $3 d-1$ & -0.00789 & 0.003072 & -0.00274 & -0.00142 & 0.004446 & 0.005713 \\
\hline $\mathrm{Cu}$ & 2 & $3 d 0$ & 0.109116 & -0.07974 & 0.044358 & -0.07079 & -0.00914 & 0.042254 \\
\hline $\mathrm{Cu}$ & 2 & $3 d 0$ & 0.005597 & -0.00545 & 0.005405 & -0.00165 & 0.001001 & -0.00047 \\
\hline $\mathrm{Cu}$ & 2 & $3 d 1$ & 0.051069 & 0.041758 & -0.05121 & 0.028305 & -0.00533 & -0.03402 \\
\hline $\mathrm{Cu}$ & 2 & $3 d 1$ & 0.002054 & 0.002713 & -0.00665 & 0.003518 & 0.000845 & -0.00082 \\
\hline $\mathrm{Cu}$ & 2 & $3 d 2$ & 0.010427 & -0.02002 & -0.00534 & 0.012231 & -0.03449 & -0.00096 \\
\hline $\mathrm{Cu}$ & 2 & $3 d 2$ & 0.000791 & -0.00160 & -0.00076 & -0.00069 & -0.00521 & -0.00240 \\
\hline $\mathrm{Cu}$ & 3 & $3 s 0$ & 0.003675 & 0.003977 & $-1.8 \mathrm{E}-05$ & 0.000023 & -0.00159 & -0.00056 \\
\hline $\mathrm{Cu}$ & 3 & $4 s 0$ & -0.08620 & 0.035221 & 0.000339 & -0.00036 & -0.00364 & -0.03323 \\
\hline
\end{tabular}




\begin{tabular}{|c|c|c|c|c|c|c|c|c|}
\hline $\mathrm{Cu}$ & 3 & $4 s 0$ & -0.00114 & -0.00639 & 0.000023 & $-3.1 \mathrm{E}-05$ & 0.009179 & 0.002077 \\
\hline $\mathrm{Cu}$ & 3 & $3 p-1$ & 0.006792 & 0.002025 & -0.00159 & 0.004306 & -0.00139 & 0.008702 \\
\hline $\mathrm{Cu}$ & 3 & $4 p-1$ & -0.03003 & -0.00898 & 0.005153 & -0.02040 & 0.005196 & -0.01672 \\
\hline $\mathrm{Cu}$ & 3 & $3 p 0$ & 0.006865 & -0.00707 & 0.000101 & 0.005842 & -0.00220 & 0.000668 \\
\hline $\mathrm{Cu}$ & 3 & $4 p 0$ & -0.03104 & 0.034730 & -0.00039 & -0.03357 & -0.00217 & -0.00997 \\
\hline $\mathrm{Cu}$ & 3 & $3 p 1$ & -0.00903 & -0.00775 & -0.0006 & -0.00677 & 0.013537 & 0.005097 \\
\hline $\mathrm{Cu}$ & 3 & $4 p 1$ & 0.035648 & 0.035334 & 0.002043 & 0.040016 & -0.05547 & -0.01728 \\
\hline $\mathrm{Cu}$ & 3 & $3 d-2$ & -0.05969 & -0.01839 & 0.107625 & 0.052102 & 0.035716 & 0.099676 \\
\hline $\mathrm{Cu}$ & 3 & $3 d-2$ & -0.00206 & -0.00148 & 0.01483 & 0.005384 & -0.00191 & 0.008858 \\
\hline $\mathrm{Cu}$ & 3 & $3 d-1$ & 0.016577 & -0.02927 & 0.047292 & -0.02499 & -0.02916 & -0.10466 \\
\hline $\mathrm{Cu}$ & 3 & $3 d-1$ & 0.000887 & -0.00262 & 0.003064 & -0.00152 & -0.00129 & -0.01057 \\
\hline $\mathrm{Cu}$ & 3 & $3 d 0$ & -0.07784 & 0.060057 & -0.00818 & -0.05559 & 0.097698 & -0.00434 \\
\hline $\mathrm{Cu}$ & 3 & $3 d 0$ & -0.00434 & 0.005546 & -0.00095 & -0.00251 & 0.016255 & -0.00105 \\
\hline $\mathrm{Cu}$ & 3 & $3 d 1$ & 0.047366 & 0.082658 & 0.018021 & 0.058999 & -0.0462 & -0.02769 \\
\hline $\mathrm{Cu}$ & 3 & $3 d 1$ & 0.001019 & 0.008178 & 0.001439 & 0.004688 & -0.01048 & -0.00463 \\
\hline $\mathrm{Cu}$ & 3 & $3 d 2$ & -0.10022 & 0.050313 & 0.073164 & -0.07148 & -0.08 & 0.04577 \\
\hline $\mathrm{Cu}$ & 3 & $3 d 2$ & -0.00695 & 0.003445 & 0.00984 & -0.00771 & -0.00736 & 0.003107 \\
\hline $\mathrm{Cu}$ & 4 & $3 s 0$ & -0.00137 & 0.005452 & -0.00037 & $-8 \mathrm{E}-06$ & 0.002565 & -0.00016 \\
\hline $\mathrm{Cu}$ & 4 & $4 s 0$ & 0.042579 & -0.00409 & 0.002502 & 0.000393 & 0.006156 & -0.00973 \\
\hline $\mathrm{Cu}$ & 4 & $4 s 0$ & 0.000053 & -0.00669 & 0.000643 & 0.000062 & -0.01519 & 0.000407 \\
\hline $\mathrm{Cu}$ & 4 & $3 p-1$ & -0.00767 & 0.003756 & -0.00205 & -0.00788 & 0.008639 & 0.009213 \\
\hline $\mathrm{Cu}$ & 4 & $4 p-1$ & 0.030869 & -0.01692 & 0.007288 & 0.026573 & -0.03238 & -0.01911 \\
\hline $\mathrm{Cu}$ & 4 & $3 p 0$ & & & & & & \\
\hline $\mathrm{Cu}$ & 4 & $4 p 0$ & 0.033692 & 0.022529 & -0.00065 & 0.067618 & 0.00336 & 0.004193 \\
\hline $\mathrm{Cu}$ & 4 & $3 p 1$ & -0.00085 & -0.00925 & 0.000039 & 0.001950 & -0.0092 & 0.003710 \\
\hline $\mathrm{Cu}$ & 4 & $4 p 1$ & 0.005991 & 0.042833 & -0.00079 & -0.00542 & 0.050537 & -0.01046 \\
\hline $\mathrm{Cu}$ & 4 & $3 d-2$ & -0.04052 & -0.03403 & 0.085616 & -0.02608 & -0.04232 & -0.04790 \\
\hline $\mathrm{Cu}$ & 4 & $3 d-2$ & -0.00320 & -0.00339 & 0.008995 & -0.00195 & -0.00319 & -0.00567 \\
\hline $\mathrm{Cu}$ & 4 & $3 d-1$ & 0.043507 & -0.02091 & 0.093193 & -0.0177 & -0.01149 & 0.139282 \\
\hline $\mathrm{Cu}$ & 4 & $3 d-1$ & 0.003355 & -0.00154 & 0.01425 & -0.00017 & -0.00518 & 0.012689 \\
\hline $\mathrm{Cu}$ & 4 & $3 d 0$ & 0.108738 & -0.08244 & 0.001871 & 0.113504 & 0.009805 & 0.027415 \\
\hline $\mathrm{Cu}$ & 4 & $3 d 0$ & 0.002444 & -0.00606 & 0.000287 & 0.004982 & 0.000720 & 0.002930 \\
\hline $\mathrm{Cu}$ & 4 & $3 d 1$ & -0.03653 & 0.059316 & 0.021668 & -0.01063 & 0.031146 & 0.041493 \\
\hline $\mathrm{Cu}$ & 4 & $3 d 1$ & -0.00342 & 0.004186 & 0.003553 & -0.00168 & 0.008954 & 0.004009 \\
\hline $\mathrm{Cu}$ & 4 & $3 d 2$ & 0.088809 & 0.046940 & 0.057463 & 0.057895 & -0.00133 & -0.01551 \\
\hline $\mathrm{Cu}$ & 4 & $3 d 2$ & 0.004408 & 0.005076 & 0.006177 & 0.002599 & -0.00060 & -0.00291 \\
\hline $\mathrm{Cu}$ & 5 & $3 s 0$ & -0.00308 & 0.006327 & -0.00511 & $-2.7 \mathrm{E}-05$ & 0.00236 & 0.002066 \\
\hline $\mathrm{Cu}$ & 5 & $4 s 0$ & 0.084802 & -0.02395 & 0.036553 & 0.000687 & 0.006621 & 0.128194 \\
\hline $\mathrm{Cu}$ & 5 & $4 s 0$ & 0.000463 & -0.00695 & 0.009954 & 0.000023 & -0.01381 & -0.00745 \\
\hline $\mathrm{Cu}$ & 5 & $3 p-1$ & -0.00166 & -0.00302 & 0.001995 & -0.00932 & -0.00735 & 0.005408 \\
\hline $\mathrm{Cu}$ & 5 & $4 p-1$ & 0.004087 & 0.01327 & -0.01074 & 0.039231 & 0.028509 & 0.000667 \\
\hline $\mathrm{Cu}$ & 5 & $3 p 0$ & -0.00397 & 0.005795 & -0.00212 & -0.01343 & -0.00122 & 0.000215 \\
\hline $\mathrm{Cu}$ & 5 & $4 p 0$ & 0.010715 & -0.02466 & 0.009526 & 0.046331 & -0.01112 & -0.00787 \\
\hline $\mathrm{Cu}$ & 5 & $3 p 1$ & -0.00033 & -0.00943 & 0.009867 & 0.000435 & -0.00797 & -0.00934 \\
\hline
\end{tabular}




\begin{tabular}{|c|c|c|c|c|c|c|c|c|}
\hline $\mathrm{Cu}$ & 5 & $4 p 1$ & 0.004540 & 0.043138 & -0.04494 & -0.00392 & 0.044234 & 0.05695 \\
\hline $\mathrm{Cu}$ & 5 & $3 d-2$ & -0.05326 & 0.045362 & 0.036907 & -0.00607 & 0.035000 & -0.02815 \\
\hline $\mathrm{Cu}$ & 5 & $3 d-2$ & -0.00253 & 0.003820 & 0.004105 & -0.00143 & 0.001854 & 0.000012 \\
\hline $\mathrm{Cu}$ & 5 & $3 d-1$ & 0.088714 & 0.006164 & -0.06118 & -0.02152 & -0.01985 & -0.11253 \\
\hline $\mathrm{Cu}$ & 5 & $3 d-1$ & 0.003302 & 0.000017 & -0.00973 & -0.00297 & -0.00628 & -0.01098 \\
\hline $\mathrm{Cu}$ & 5 & $3 d 0$ & -0.13986 & -0.05125 & 0.027482 & -0.04264 & 0.024399 & -0.01960 \\
\hline $\mathrm{Cu}$ & 5 & $3 d 0$ & -0.00632 & -0.00465 & 0.003265 & -0.00092 & 0.001136 & -0.00512 \\
\hline $\mathrm{Cu}$ & 5 & $3 d 1$ & -0.11551 & -0.05031 & 0.05104 & 0.012775 & -0.05426 & 0.032691 \\
\hline $\mathrm{Cu}$ & 5 & $3 d 1$ & -0.00807 & -0.00325 & 0.006631 & 0.000292 & -0.01244 & 0.000928 \\
\hline $\mathrm{Cu}$ & 5 & $3 d 2$ & -0.05161 & 0.062769 & -0.08256 & -0.02168 & -0.00342 & 0.028938 \\
\hline $\mathrm{Cu}$ & 5 & $3 d 2$ & $-5 \mathrm{E}-06$ & 0.005442 & -0.00857 & 0.000138 & -0.00185 & 0.008361 \\
\hline $\mathrm{Cu}$ & 6 & $3 s 0$ & 0.000158 & 0.004986 & 0.00491 & 0.000062 & -0.00325 & -0.00247 \\
\hline $\mathrm{Cu}$ & 6 & $4 s 0$ & 0.00284 & 0.007467 & -0.03549 & -0.00013 & -0.00734 & -0.15340 \\
\hline $\mathrm{Cu}$ & 6 & $4 s 0$ & -0.00032 & -0.00654 & -0.00947 & -0.00013 & 0.018886 & 0.009023 \\
\hline $\mathrm{Cu}$ & 6 & $3 p-1$ & -0.00540 & 0.007396 & 0.004581 & -0.0024 & -0.01699 & -0.00022 \\
\hline $\mathrm{Cu}$ & 6 & $4 p-1$ & 0.022950 & -0.03264 & -0.02261 & 0.023422 & 0.072017 & 0.029221 \\
\hline $\mathrm{Cu}$ & 6 & $3 p 0$ & -0.00480 & -0.00772 & -0.00872 & -0.00199 & 0.003397 & 0.010455 \\
\hline $\mathrm{Cu}$ & 6 & $4 p 0$ & 0.020804 & 0.037274 & 0.038968 & 0.018384 & -0.03479 & -0.06045 \\
\hline $\mathrm{Cu}$ & 6 & $3 p 1$ & 0.010363 & $-2.6 \mathrm{E}-05$ & -0.00078 & 0.013131 & 0.000137 & 0.003117 \\
\hline $\mathrm{Cu}$ & 6 & $4 p 1$ & & & & & & -0.00713 \\
\hline $\mathrm{Cu}$ & 6 & $3 d-2$ & 0.182802 & -0.02937 & -0.04645 & 0.001862 & 0.005384 & -0.03513 \\
\hline $\mathrm{Cu}$ & 6 & $3 d-2$ & 0.009801 & -0.00158 & -0.00647 & 0.000637 & 0.001539 & -0.00289 \\
\hline $\mathrm{Cu}$ & 6 & $3 d-1$ & 0.041282 & -0.06269 & 0.003927 & 0.060957 & 0.044973 & 0.056865 \\
\hline $\mathrm{Cu}$ & 6 & $3 d-1$ & 0.002075 & -0.00669 & 0.002164 & 0.004411 & 0.009518 & 0.012308 \\
\hline $\mathrm{Cu}$ & 6 & $3 d 0$ & 0.056901 & 0.076836 & 0.113576 & 0.047085 & 0.062983 & 0.010804 \\
\hline $\mathrm{Cu}$ & 6 & $3 d 0$ & 0.002934 & 0.006244 & 0.013516 & 0.002295 & 0.009028 & -0.00379 \\
\hline $\mathrm{Cu}$ & 6 & $3 d 1$ & 0.044809 & -0.00918 & 0.018510 & -0.01852 & -0.01000 & -0.03475 \\
\hline $\mathrm{Cu}$ & 6 & $3 d 1$ & 0.002416 & -0.00010 & 0.001189 & 0.001406 & -0.00145 & -0.00375 \\
\hline $\mathrm{Cu}$ & 6 & $3 d 2$ & -0.04884 & -0.06979 & -0.01767 & -0.09451 & 0.071803 & -0.07658 \\
\hline $\mathrm{Cu}$ & 6 & $3 d 2$ & -0.00324 & -0.00425 & -0.00427 & -0.00853 & 0.003589 & -0.00498 \\
\hline $\mathrm{Cu}$ & 7 & $3 s 0$ & 0.002550 & 0.006787 & 0.007645 & 0.000019 & 0.000619 & -0.00324 \\
\hline $\mathrm{Cu}$ & 7 & $4 s 0$ & -0.05900 & -0.03659 & -0.05416 & 0.000006 & 0.00205 & -0.19828 \\
\hline $\mathrm{Cu}$ & 7 & $4 s 0$ & -0.00090 & -0.00703 & -0.01474 & 0.000001 & -0.00375 & 0.011749 \\
\hline $\mathrm{Cu}$ & 7 & $3 p-1$ & 0.001752 & 0.010216 & 0.014831 & 0.002914 & 0.003673 & -0.01083 \\
\hline $\mathrm{Cu}$ & 7 & $4 p-1$ & -0.00516 & -0.04593 & -0.06829 & -0.00784 & -0.01511 & 0.083820 \\
\hline $\mathrm{Cu}$ & 7 & $3 p 0$ & 0.005565 & -0.00442 & -0.00291 & 0.015555 & 0.005090 & 0.001944 \\
\hline $\mathrm{Cu}$ & 7 & $4 p 0$ & -0.02148 & 0.019434 & 0.013383 & -0.04308 & -0.00200 & -0.01629 \\
\hline $\mathrm{Cu}$ & 7 & $3 p 1$ & -0.01387 & 0.001609 & -0.00084 & -0.00881 & 0.001435 & 0.003312 \\
\hline $\mathrm{Cu}$ & 7 & $4 p 1$ & 0.058640 & -0.00623 & 0.003104 & 0.035979 & -0.00337 & -0.00792 \\
\hline $\mathrm{Cu}$ & 7 & $3 d-2$ & -0.03875 & 0.000914 & -0.03824 & -0.00885 & 0.003807 & 0.016527 \\
\hline $\mathrm{Cu}$ & 7 & $3 d-2$ & -0.00066 & -0.00040 & -0.00402 & 0.000971 & -0.00015 & 0.002298 \\
\hline $\mathrm{Cu}$ & 7 & $3 d-1$ & -0.00154 & -0.05035 & -0.04489 & -0.01033 & -0.04713 & 0.010119 \\
\hline $\mathrm{Cu}$ & 7 & $3 d-1$ & -0.00059 & -0.00350 & -0.00498 & -0.00123 & -0.01352 & 0.004573 \\
\hline $\mathrm{Cu}$ & 7 & $3 d 0$ & -0.02795 & -0.06243 & -0.04149 & -0.10957 & 0.031677 & 0.015228 \\
\hline
\end{tabular}




\begin{tabular}{|c|c|c|c|c|c|c|c|c|}
\hline $\mathrm{Cu}$ & 7 & $3 d 0$ & 0.000283 & -0.00471 & -0.00482 & -0.00580 & 0.002911 & 0.006495 \\
\hline $\mathrm{Cu}$ & 7 & $3 d 1$ & 0.110305 & 0.009092 & 0.034657 & 0.011985 & -0.00059 & 0.044593 \\
\hline $\mathrm{Cu}$ & 7 & $3 d 1$ & 0.006702 & 0.000301 & 0.005300 & 0.002099 & $-6.9 \mathrm{E}-05$ & 0.004027 \\
\hline $\mathrm{Cu}$ & 7 & $3 d 2$ & 0.029121 & -0.06501 & -0.06180 & 0.062728 & -0.00621 & 0.016732 \\
\hline $\mathrm{Cu}$ & 7 & $3 d 2$ & 0.002282 & -0.00581 & -0.00621 & 0.003589 & 0.002523 & 0.010920 \\
\hline $\mathrm{Cu}$ & 8 & $3 s 0$ & -0.00142 & 0.006175 & 0.004377 & -0.00005 & 0.001820 & -0.00174 \\
\hline $\mathrm{Cu}$ & 8 & $4 s 0$ & 0.042902 & -0.02110 & -0.03120 & 0.000392 & 0.005060 & -0.10816 \\
\hline $\mathrm{Cu}$ & 8 & $4 s 0$ & 0.000059 & -0.00688 & -0.00845 & 0.000064 & -0.01067 & 0.006481 \\
\hline $\mathrm{Cu}$ & 8 & $3 p-1$ & 0.008641 & 0.007043 & 0.005972 & 0.006758 & 0.006093 & 0.000603 \\
\hline $\mathrm{Cu}$ & 8 & $4 p-1$ & -0.03414 & -0.03222 & -0.02857 & -0.03231 & -0.03377 & 0.025195 \\
\hline $\mathrm{Cu}$ & 8 & $3 p 0$ & -0.00109 & 0.005356 & 0.001895 & -0.00139 & -0.00820 & -0.00423 \\
\hline $\mathrm{Cu}$ & 8 & $4 p 0$ & 0.002350 & -0.02321 & -0.00829 & 0.001439 & 0.012327 & 0.015343 \\
\hline $\mathrm{Cu}$ & 8 & $3 p 1$ & 0.010672 & -0.00724 & -0.00618 & 0.008703 & -0.00688 & 0.009807 \\
\hline $\mathrm{Cu}$ & 8 & $4 p 1$ & -0.03982 & 0.032907 & 0.027108 & -0.04321 & 0.02718 & -0.04132 \\
\hline $\mathrm{Cu}$ & 8 & $3 d-2$ & 0.024870 & -0.06940 & -0.05615 & 0.016394 & -0.01421 & 0.044812 \\
\hline $\mathrm{Cu}$ & 8 & $3 d-2$ & -0.00076 & -0.00622 & -0.00565 & 0.000380 & -0.00042 & 0.009497 \\
\hline $\mathrm{Cu}$ & 8 & $3 d-1$ & -0.03054 & 0.024202 & -0.02162 & 0.049611 & 0.000136 & -0.07091 \\
\hline $\mathrm{Cu}$ & 8 & $3 d-1$ & 0.000222 & 0.001860 & -0.00436 & 0.00447 & 0.005841 & -0.00843 \\
\hline $\mathrm{Cu}$ & 8 & $3 d 0$ & -0.05359 & -0.06115 & -0.02402 & -0.02456 & -0.00054 & -0.03605 \\
\hline $\mathrm{Cu}$ & 8 & $3 d 0$ & -0.00267 & -0.00500 & -0.00301 & -0.00173 & 0.001300 & -0.00080 \\
\hline $\mathrm{Cu}$ & 8 & $3 d 1$ & -0.09566 & -0.05095 & -0.08333 & 0.056768 & -0.00396 & -0.09337 \\
\hline $\mathrm{Cu}$ & 8 & $3 d 1$ & -0.00423 & -0.00324 & -0.01198 & 0.005436 & -0.00271 & -0.00704 \\
\hline $\mathrm{Cu}$ & 8 & $3 d 2$ & & & & & & \\
\hline $\mathrm{Cu}$ & 8 & $3 d 2$ & 0.002301 & -0.00157 & 0.007871 & 0.002567 & -0.00470 & -0.00260 \\
\hline $\mathrm{Cu}$ & 9 & $3 s 0$ & -0.00551 & 0.005328 & 0.003358 & 0.000040 & -0.00229 & -0.00170 \\
\hline $\mathrm{Cu}$ & 9 & $4 s 0$ & 0.147357 & -0.00056 & -0.02404 & -0.00039 & -0.00544 & -0.10573 \\
\hline $\mathrm{Cu}$ & 9 & $4 s 0$ & 0.001061 & -0.00667 & -0.00648 & -0.00014 & 0.013467 & 0.006442 \\
\hline $\mathrm{Cu}$ & 9 & $3 p-1$ & 0.005349 & 0.004276 & -0.00029 & 0.028248 & -0.00209 & 0.007317 \\
\hline $\mathrm{Cu}$ & 9 & $4 p-1$ & -0.02134 & -0.01797 & -0.00072 & -0.10569 & 0.010036 & -0.00933 \\
\hline $\mathrm{Cu}$ & 9 & $3 p 0$ & -0.00325 & -0.00654 & -0.00590 & 0.005406 & 0.001284 & 0.006810 \\
\hline $\mathrm{Cu}$ & 9 & $4 p 0$ & 0.016157 & 0.031993 & 0.026346 & -0.02482 & -0.01811 & -0.04139 \\
\hline $\mathrm{Cu}$ & 9 & $3 p 1$ & -0.00671 & 0.008096 & 0.003581 & -0.00167 & -0.01362 & -0.00359 \\
\hline $\mathrm{Cu}$ & 9 & $4 p 1$ & 0.022085 & -0.03558 & -0.01688 & -0.00028 & 0.060137 & 0.026994 \\
\hline $\mathrm{Cu}$ & 9 & $3 d-2$ & -0.05217 & 0.034669 & -0.07350 & -0.02994 & -0.04082 & -0.04401 \\
\hline $\mathrm{Cu}$ & 9 & $3 d-2$ & -0.00108 & 0.002048 & -0.00930 & -0.00252 & 0.000404 & -0.00439 \\
\hline $\mathrm{Cu}$ & 9 & $3 d-1$ & -0.05057 & -0.02605 & 0.038267 & -0.06099 & -0.01922 & -0.07171 \\
\hline $\mathrm{Cu}$ & 9 & $3 d-1$ & -0.00170 & -0.00208 & 0.002968 & 0.000556 & 0.00005 & -0.00621 \\
\hline $\mathrm{Cu}$ & 9 & $3 d 0$ & -0.00333 & 0.062339 & 0.074694 & -0.02433 & 0.086498 & 0.005431 \\
\hline $\mathrm{Cu}$ & 9 & $3 d 0$ & 0.000714 & 0.005445 & 0.008987 & -0.00087 & 0.015012 & -0.00267 \\
\hline $\mathrm{Cu}$ & 9 & $3 d 1$ & -0.05139 & -0.06620 & -0.01193 & -0.00449 & 0.048089 & 0.060428 \\
\hline $\mathrm{Cu}$ & 9 & $3 d 1$ & -0.00623 & -0.00668 & 0.000360 & -0.00239 & 0.010788 & 0.010702 \\
\hline $\mathrm{Cu}$ & 9 & $3 d 2$ & 0.003058 & 0.049070 & 0.071483 & -0.00932 & -0.08011 & 0.085765 \\
\hline $\mathrm{Cu}$ & 9 & $3 d 2$ & 0.002273 & 0.002877 & 0.010755 & -0.00328 & -0.00714 & 0.006206 \\
\hline $\mathrm{Cu}$ & 10 & $3 s 0$ & -0.00308 & 0.006322 & 0.005113 & 0.000025 & 0.002361 & -0.00205 \\
\hline
\end{tabular}




\begin{tabular}{|c|c|c|c|c|c|c|c|c|}
\hline $\mathrm{Cu}$ & 10 & $4 s 0$ & 0.084780 & -0.02407 & -0.03666 & -0.00055 & 0.006866 & -0.12783 \\
\hline $\mathrm{Cu}$ & 10 & $4 s 0$ & 0.000474 & -0.00694 & -0.00993 & $-2.3 \mathrm{E}-05$ & -0.0138 & 0.007416 \\
\hline $\mathrm{Cu}$ & 10 & $3 p-1$ & 0.001688 & 0.003035 & 0.001975 & -0.00931 & 0.007321 & 0.00538 \\
\hline $\mathrm{Cu}$ & 10 & $4 p-1$ & -0.00417 & -0.0133 & -0.01061 & 0.039188 & & 0.000735 \\
\hline $\mathrm{Cu}$ & 10 & $3 p 0$ & 0.003987 & -0.00579 & -0.00212 & -0.01342 & 0.001153 & 0.000213 \\
\hline $\mathrm{Cu}$ & 10 & $4 p 0$ & -0.01074 & 0.024666 & 0.009471 & 0.046235 & 0.011328 & -0.00782 \\
\hline $\mathrm{Cu}$ & 10 & $3 p 1$ & 0.000319 & 0.009423 & 0.009866 & 0.000437 & 0.007965 & -0.0093 \\
\hline $\mathrm{Cu}$ & 10 & $4 p 1$ & -0.00447 & -0.04312 & -0.04496 & -0.00395 & -0.04425 & 0.056755 \\
\hline $\mathrm{Cu}$ & 10 & $3 d-2$ & -0.05326 & 0.045182 & -0.03702 & 0.006204 & 0.034971 & 0.028109 \\
\hline $\mathrm{Cu}$ & 10 & $3 d-2$ & -0.00254 & 0.003796 & -0.00412 & 0.001438 & 0.001841 & $-1.2 \mathrm{E}-05$ \\
\hline $\mathrm{Cu}$ & 10 & $3 d-1$ & 0.088449 & 0.006154 & 0.061357 & 0.021586 & -0.02018 & 0.112395 \\
\hline $\mathrm{Cu}$ & 10 & $3 d-1$ & 0.003295 & 0.000031 & 0.009756 & 0.002972 & -0.0063 & 0.010954 \\
\hline $\mathrm{Cu}$ & 10 & $3 d 0$ & -0.13993 & -0.05129 & -0.0274 & 0.042782 & 0.024824 & 0.01976 \\
\hline $\mathrm{Cu}$ & 10 & $3 d 0$ & -0.00632 & -0.00465 & -0.00326 & 0.000915 & 0.00116 & 0.005115 \\
\hline $\mathrm{Cu}$ & 10 & $3 d 1$ & -0.11544 & -0.05025 & -0.05095 & -0.01273 & -0.05425 & -0.033 \\
\hline $\mathrm{Cu}$ & 10 & $3 d 1$ & -0.00807 & -0.00325 & -0.00662 & -0.00029 & -0.01 & -0.00099 \\
\hline $\mathrm{Cu}$ & 10 & $3 d 2$ & -0.05187 & 0.062702 & 0.082595 & 0.021771 & -0.00318 & -0.02885 \\
\hline $\mathrm{Cu}$ & 10 & $3 d 2$ & $-2.2 \mathrm{E}-05$ & 0.005443 & 0.00857 & -0.00014 & -0.00182 & -0.00837 \\
\hline $\mathrm{Cu}$ & 11 & $3 s 0$ & 0.004356 & 0.006527 & 0.007783 & $-9 \mathrm{E}-06$ & 0.002074 & -0.00294 \\
\hline $\mathrm{Cu}$ & 11 & & & -0.02974 & & & & \\
\hline $\mathrm{Cu}$ & 11 & $4 s 0$ & -0.00135 & -0.00697 & -0.01502 & 0.000068 & -0.01214 & 0.010745 \\
\hline $\mathrm{Cu}$ & 11 & $3 p-1$ & -0.00447 & 0.009122 & 0.012079 & -0.00072 & 0.008768 & -0.00571 \\
\hline $\mathrm{Cu}$ & 11 & $4 p-1$ & 0.021642 & -0.04059 & -0.05596 & -0.00213 & -0.04353 & 0.057562 \\
\hline $\mathrm{Cu}$ & 11 & $3 p 0$ & 0.000425 & 0.003652 & 0.003038 & 0.021191 & -0.00939 & -0.00572 \\
\hline $\mathrm{Cu}$ & 11 & $4 p 0$ & 0.002457 & -0.01696 & -0.01364 & -0.07244 & 0.016319 & 0.022772 \\
\hline $\mathrm{Cu}$ & 11 & $3 p 1$ & 0.008108 & 0.004993 & 0.00909 & -0.00589 & 0.005278 & -0.00795 \\
\hline $\mathrm{Cu}$ & 11 & $4 p 1$ & -0.03354 & -0.02313 & -0.04143 & 0.026701 & -0.02448 & 0.049234 \\
\hline $\mathrm{Cu}$ & 11 & $3 d-2$ & 0.065553 & 0.052135 & 0.065957 & 0.031924 & 0.01295 & -0.05038 \\
\hline $\mathrm{Cu}$ & 11 & $3 d-2$ & 0.001251 & 0.005298 & 0.006503 & 0.001501 & 0.001202 & -0.01291 \\
\hline $\mathrm{Cu}$ & 11 & $3 d-1$ & -0.12024 & 0.040244 & 0.029536 & 0.032015 & -0.01082 & -0.02868 \\
\hline $\mathrm{Cu}$ & 11 & $3 d-1$ & -0.0079 & 0.003075 & 0.002745 & 0.001433 & 0.004446 & -0.00572 \\
\hline $\mathrm{Cu}$ & 11 & $3 d 0$ & 0.109274 & -0.07976 & -0.04424 & 0.070867 & -0.00951 & -0.04223 \\
\hline $\mathrm{Cu}$ & 11 & $3 d 0$ & 0.005617 & -0.00545 & -0.0054 & 0.001645 & 0.000973 & 0.00048 \\
\hline $\mathrm{Cu}$ & 11 & $3 d 1$ & 0.050935 & 0.041817 & 0.051191 & -0.0285 & -0.00549 & 0.033988 \\
\hline $\mathrm{Cu}$ & 11 & $3 d 1$ & 0.002056 & 0.002713 & 0.00664 & -0.00354 & 0.000819 & 0.000802 \\
\hline $\mathrm{Cu}$ & 11 & $3 d 2$ & 0.010219 & -0.02007 & 0.005118 & -0.01233 & -0.03443 & 0.000658 \\
\hline $\mathrm{Cu}$ & 11 & $3 d 2$ & 0.000775 & -0.00161 & 0.000739 & 0.000675 & -0.00522 & 0.002381 \\
\hline $\mathrm{Cu}$ & 12 & $3 s 0$ & -0.00204 & 0.004899 & -0.0025 & 0.00005 & -0.00143 & 0.000577 \\
\hline $\mathrm{Cu}$ & 12 & $4 s 0$ & 0.058697 & 0.009643 & 0.017729 & 0.000129 & -0.00359 & 0.036407 \\
\hline $\mathrm{Cu}$ & 12 & $4 s 0$ & 0.000224 & -0.00656 & 0.00484 & $-8.7 \mathrm{E}-05$ & 0.008376 & -0.00218 \\
\hline $\mathrm{Cu}$ & 12 & $3 p-1$ & -0.00058 & -0.00817 & 0.000952 & -0.01807 & 0.00996 & 0.007747 \\
\hline $\mathrm{Cu}$ & 12 & $4 p-1$ & 0.000212 & 0.035749 & -0.00626 & 0.073068 & -0.0409 & -0.01134 \\
\hline $\mathrm{Cu}$ & 12 & $3 p 0$ & -0.00108 & -0.00679 & 0.00451 & -0.00089 & -0.00435 & -0.00541 \\
\hline $\mathrm{Cu}$ & 12 & $4 p 0$ & 0.005632 & 0.033246 & -0.02003 & 0.000982 & 0.003503 & 0.020835 \\
\hline
\end{tabular}




\begin{tabular}{|c|c|c|c|c|c|c|c|c|}
\hline $\mathrm{Cu}$ & 12 & $3 p 1$ & -0.00251 & 0.003397 & -0.00263 & -0.02614 & -0.01186 & 0.00448 \\
\hline $\mathrm{Cu}$ & 12 & $4 p 1$ & 0.003773 & -0.01538 & 0.011073 & 0.09977 & 0.039326 & -0.014 \\
\hline $\mathrm{Cu}$ & 12 & $3 d-2$ & -0.04527 & -0.05286 & 0.040394 & 0.028837 & 0.082168 & 0.099258 \\
\hline $\mathrm{Cu}$ & 12 & $3 d-2$ & -0.00347 & -0.00329 & 0.006636 & & 0.003086 & 0.008786 \\
\hline $\mathrm{Cu}$ & 12 & $3 d-1$ & 0.08086 & 0.05814 & 0.02913 & 0.03332 & -0.02822 & -0.01887 \\
\hline $\mathrm{Cu}$ & 12 & $3 d-1$ & 0.003518 & 0.005713 & 0.002931 & -0.00166 & -0.00804 & -0.00128 \\
\hline $\mathrm{Cu}$ & 12 & $3 d 0$ & -0.00864 & 0.063248 & -0.06882 & -0.00916 & 0.094475 & -0.01516 \\
\hline $\mathrm{Cu}$ & 12 & $3 d 0$ & $-4.3 \mathrm{E}-05$ & 0.005508 & -0.00827 & -0.00052 & 0.013987 & 0.000414 \\
\hline $\mathrm{Cu}$ & 12 & $3 d 1$ & 0.047158 & -0.04571 & 0.029485 & 0.020644 & 0.017744 & -0.09807 \\
\hline $\mathrm{Cu}$ & 12 & $3 d 1$ & -0.00068 & -0.00465 & 0.001084 & -0.00388 & 0.003516 & -0.01089 \\
\hline $\mathrm{Cu}$ & 12 & $3 d 2$ & 0.213482 & -0.03508 & -0.1025 & -0.01335 & 0.021904 & -0.06039 \\
\hline $\mathrm{Cu}$ & 12 & $3 d 2$ & 0.010976 & -0.00209 & -0.01367 & -0.00221 & 0.002272 & -0.00481 \\
\hline $\mathrm{Cu}$ & 13 & $3 s 0$ & -0.00143 & 0.006179 & -0.00438 & 0.000043 & 0.001823 & 0.001738 \\
\hline $\mathrm{Cu}$ & 13 & $4 s 0$ & 0.042863 & -0.02103 & 0.031167 & -0.00023 & 0.004987 & 0.108317 \\
\hline $\mathrm{Cu}$ & 13 & $4 s 0$ & 0.000069 & -0.00688 & 0.008454 & $-5.8 \mathrm{E}-05$ & -0.01063 & -0.00646 \\
\hline $\mathrm{Cu}$ & 13 & $3 p-1$ & -0.00863 & -0.00704 & 0.005977 & 0.006763 & -0.00607 & 0.000624 \\
\hline $\mathrm{Cu}$ & 13 & $4 p-1$ & 0.034106 & 0.032176 & -0.02859 & -0.03231 & 0.033625 & 0.025083 \\
\hline $\mathrm{Cu}$ & 13 & $3 p 0$ & 0.001079 & -0.00535 & 0.001891 & -0.00141 & 0.008168 & -0.00423 \\
\hline $\mathrm{Cu}$ & 13 & $4 p 0$ & -0.00231 & 0.023176 & -0.00828 & 0.001509 & -0.01228 & 0.015314 \\
\hline $\mathrm{Cu}$ & 13 & $3 p 1$ & -0.01068 & 0.007246 & -0.00618 & 0.008704 & 0.006853 & 0.009811 \\
\hline $\mathrm{Cu}$ & 13 & $4 p 1$ & 0.039851 & -0.03293 & 0.027089 & -0.04321 & -0.02706 & -0.04131 \\
\hline $\mathrm{Cu}$ & 13 & $3 d-2$ & 0.025093 & -0.06949 & 0.056136 & -0.01645 & -0.01429 & -0.04476 \\
\hline $\mathrm{Cu}$ & 13 & $3 d-2$ & -0.00074 & -0.00623 & 0.005659 & -0.00039 & -0.0004 & -0.00947 \\
\hline $\mathrm{Cu}$ & 13 & $3 d-1$ & -0.03044 & 0.02419 & 0.021447 & -0.04954 & 0.000034 & 0.071041 \\
\hline $\mathrm{Cu}$ & 13 & $3 d-1$ & 0.000231 & 0.001859 & 0.004343 & -0.00447 & 0.005842 & 0.008453 \\
\hline $\mathrm{Cu}$ & 13 & $3 d 0$ & -0.05368 & -0.06124 & 0.024152 & 0.024647 & -0.00018 & 0.036083 \\
\hline $\mathrm{Cu}$ & 13 & $3 d 0$ & -0.00269 & -0.00501 & 0.003003 & 0.001745 & 0.001328 & 0.000803 \\
\hline $\mathrm{Cu}$ & 13 & $3 d 1$ & -0.09578 & -0.05109 & 0.083363 & -0.0567 & -0.00422 & 0.093269 \\
\hline $\mathrm{Cu}$ & 13 & $3 d 1$ & -0.00424 & -0.00324 & 0.011976 & -0.00543 & -0.00273 & 0.007022 \\
\hline $\mathrm{Cu}$ & 13 & $3 d 2$ & -0.01014 & -0.015 & -0.07322 & 0.000485 & -0.02 & 0.029061 \\
\hline $\mathrm{Cu}$ & 13 & $3 d 2$ & 0.0023 & -0.00158 & -0.00786 & -0.00257 & -0.00471 & 0.002588 \\
\hline $\mathrm{Cu}$ & 14 & $3 s 0$ & -0.00137 & 0.005457 & 0.000365 & 0.000004 & 0.002561 & 0.000163 \\
\hline $\mathrm{Cu}$ & 14 & $4 s 0$ & 0.042434 & -0.004 & -0.00229 & -0.00033 & 0.006004 & 0.009632 \\
\hline $\mathrm{Cu}$ & 14 & $4 s 0$ & 0.000066 & -0.0067 & -0.00067 & $-6.2 \mathrm{E}-05$ & -0.01521 & -0.00044 \\
\hline $\mathrm{Cu}$ & 14 & $3 p-1$ & 0.00769 & -0.00377 & -0.00205 & -0.00788 & -0.00867 & 0.009157 \\
\hline $\mathrm{Cu}$ & 14 & $4 p-1$ & -0.03091 & 0.016966 & 0.007307 & 0.026634 & 0.03241 & -0.01889 \\
\hline $\mathrm{Cu}$ & 14 & $3 p 0$ & 0.009379 & 0.004947 & 0.000181 & -0.02183 & -0.0036 & -0.00211 \\
\hline $\mathrm{Cu}$ & 14 & $4 p 0$ & -0.03372 & -0.02256 & -0.00071 & 0.067631 & -0.00325 & 0.004178 \\
\hline $\mathrm{Cu}$ & 14 & $3 p 1$ & 0.000842 & 0.009235 & 0.000046 & 0.001951 & 0.009198 & 0.003713 \\
\hline $\mathrm{Cu}$ & 14 & $4 p 1$ & -0.00595 & -0.04278 & -0.00083 & -0.00543 & -0.05056 & -0.01052 \\
\hline $\mathrm{Cu}$ & 14 & $3 d-2$ & -0.04031 & -0.03413 & -0.08569 & 0.025946 & -0.0423 & 0.047653 \\
\hline $\mathrm{Cu}$ & 14 & $3 d-2$ & -0.00319 & -0.0034 & -0.00901 & 0.001939 & -0.00319 & 0.005622 \\
\hline $\mathrm{Cu}$ & 14 & $3 d-1$ & 0.04359 & -0.02093 & -0.09332 & 0.017493 & -0.01113 & -0.13917 \\
\hline $\mathrm{Cu}$ & 14 & $3 d-1$ & 0.003355 & -0.00153 & -0.01425 & 0.000164 & -0.00512 & -0.01267 \\
\hline
\end{tabular}




\begin{tabular}{|c|c|c|c|c|c|c|c|c|}
\hline $\mathrm{Cu}$ & 14 & $3 d 0$ & 0.108693 & -0.08257 & -0.00192 & -0.11344 & 0.009363 & -0.02748 \\
\hline $\mathrm{Cu}$ & 14 & $3 d 0$ & 0.002441 & -0.00607 & -0.00029 & -0.00498 & 0.000683 & -0.00292 \\
\hline $\mathrm{Cu}$ & 14 & $3 d 1$ & -0.03654 & 0.059369 & -0.02151 & 0.010414 & 0.031041 & -0.04168 \\
\hline $\mathrm{Cu}$ & 14 & $3 d 1$ & -0.00343 & 0.004186 & -0.00353 & 0.001667 & 0.008925 & -0.00404 \\
\hline $\mathrm{Cu}$ & 14 & $3 d 2$ & 0.08872 & 0.046915 & -0.05742 & -0.05785 & -0.00141 & 0.015434 \\
\hline $\mathrm{Cu}$ & 14 & $3 d 2$ & 0.004398 & 0.005082 & -0.00616 & -0.0026 & -0.0006 & 0.00291 \\
\hline $\mathrm{Cu}$ & 15 & $3 s 0$ & 0.002544 & 0.006785 & -0.00765 & $-2.3 \mathrm{E}-05$ & 0.000614 & 0.00324 \\
\hline $\mathrm{Cu}$ & 15 & $4 s 0$ & -0.0589 & -0.03664 & 0.054195 & -0.00016 & 0.001443 & 0.198282 \\
\hline $\mathrm{Cu}$ & 15 & $4 s 0$ & -0.0009 & -0.00702 & 0.014738 & 0.000012 & -0.00373 & -0.01174 \\
\hline $\mathrm{Cu}$ & 15 & $3 p-1$ & -0.00175 & -0.01022 & 0.014828 & 0.002924 & -0.00367 & -0.01083 \\
\hline $\mathrm{Cu}$ & 15 & $4 p-1$ & 0.005149 & 0.04593 & -0.06828 & -0.00787 & 0.014984 & 0.083804 \\
\hline $\mathrm{Cu}$ & 15 & $3 p 0$ & -0.00557 & 0.004414 & -0.00294 & 0.015563 & -0.00513 & 0.001924 \\
\hline $\mathrm{Cu}$ & 15 & $4 p 0$ & 0.021493 & -0.01942 & 0.013471 & -0.04309 & 0.002126 & -0.01624 \\
\hline $\mathrm{Cu}$ & 15 & $3 p 1$ & 0.013867 & -0.00162 & -0.00087 & -0.0088 & -0.00144 & 0.003261 \\
\hline $\mathrm{Cu}$ & 15 & $4 p 1$ & -0.05864 & 0.00626 & 0.003207 & 0.035947 & 0.003403 & -0.00778 \\
\hline $\mathrm{Cu}$ & 15 & $3 d-2$ & -0.03866 & 0.000852 & 0.038337 & 0.008782 & 0.003861 & -0.01654 \\
\hline $\mathrm{Cu}$ & 15 & $3 d-2$ & -0.00065 & -0.00041 & 0.004024 & -0.00098 & -0.00015 & -0.00234 \\
\hline $\mathrm{Cu}$ & 15 & $3 d-1$ & -0.00149 & -0.05031 & 0.044913 & 0.010437 & -0.047 & -0.01014 \\
\hline $\mathrm{Cu}$ & 15 & $3 d-1$ & & & & & & \\
\hline $\mathrm{Cu}$ & 15 & $3 d 0$ & -0.02815 & -0.06233 & 0.041328 & 0.109601 & 0.03132 & -0.01529 \\
\hline $\mathrm{Cu}$ & 15 & $3 d 0$ & 0.000266 & -0.00471 & 0.004824 & 0.005804 & 0.002901 & -0.00651 \\
\hline $\mathrm{Cu}$ & 15 & $3 d 1$ & 0.110347 & 0.009061 & -0.03451 & -0.0118 & -0.00051 & -0.04443 \\
\hline $\mathrm{Cu}$ & 15 & $3 d 1$ & 0.006712 & 0.000305 & -0.0053 & -0.0021 & $-5.9 \mathrm{E}-05$ & -0.004 \\
\hline $\mathrm{Cu}$ & 15 & $3 d 2$ & 0.029147 & -0.06496 & 0.061809 & -0.06273 & -0.006 & -0.01666 \\
\hline $\mathrm{Cu}$ & 15 & $3 d 2$ & 0.002284 & -0.0058 & 0.00621 & -0.00358 & 0.002565 & -0.0109 \\
\hline $\mathrm{Cu}$ & 16 & $3 s 0$ & 0.000165 & 0.004984 & & $-6.3 \mathrm{E}-05$ & & 0.00247 \\
\hline $\mathrm{Cu}$ & 16 & $4 s 0$ & 0.002863 & 0.007346 & 0.035627 & 0.000227 & -0.00703 & 0.153469 \\
\hline $\mathrm{Cu}$ & 16 & $4 s 0$ & -0.00033 & -0.00653 & 0.009472 & 0.000136 & 0.018872 & -0.00899 \\
\hline $\mathrm{Cu}$ & 16 & $3 p-1$ & 0.005389 & -0.00739 & 0.004603 & -0.00239 & 0.01698 & -0.0002 \\
\hline $\mathrm{Cu}$ & 16 & $4 p-1$ & -0.02291 & 0.032649 & -0.02269 & 0.023427 & -0.07201 & 0.029167 \\
\hline $\mathrm{Cu}$ & 16 & $3 p 0$ & 0.004807 & 0.007724 & -0.00872 & -0.00198 & -0.00338 & 0.010459 \\
\hline $\mathrm{Cu}$ & 16 & $4 p 0$ & -0.02086 & -0.0373 & 0.038947 & 0.018385 & 0.034646 & -0.06045 \\
\hline $\mathrm{Cu}$ & 16 & $3 p 1$ & -0.01034 & 0.000027 & -0.00081 & 0.013131 & -0.00015 & 0.003126 \\
\hline $\mathrm{Cu}$ & 16 & $4 p 1$ & 0.046374 & -0.00093 & 0.002802 & -0.05064 & 0.002122 & -0.00712 \\
\hline $\mathrm{Cu}$ & 16 & $3 d-2$ & 0.18249 & -0.02953 & 0.046409 & -0.00179 & 0.00547 & 0.035087 \\
\hline $\mathrm{Cu}$ & 16 & $3 d-2$ & 0.009794 & -0.0016 & 0.006478 & -0.00064 & 0.001543 & 0.002894 \\
\hline $\mathrm{Cu}$ & 16 & $3 d-1$ & 0.041167 & -0.06251 & -0.00398 & -0.06103 & 0.045072 & -0.05682 \\
\hline $\mathrm{Cu}$ & 16 & $3 d-1$ & 0.002066 & -0.00669 & -0.00217 & -0.00442 & 0.009523 & -0.0123 \\
\hline $\mathrm{Cu}$ & 16 & $3 d 0$ & 0.056821 & 0.07683 & -0.1136 & -0.04717 & 0.06328 & -0.01078 \\
\hline $\mathrm{Cu}$ & 16 & $3 d 0$ & 0.002929 & 0.006233 & -0.01352 & -0.0023 & 0.009078 & 0.003787 \\
\hline $\mathrm{Cu}$ & 16 & $3 d 1$ & 0.044961 & -0.00916 & -0.01864 & 0.018509 & -0.01008 & 0.034576 \\
\hline $\mathrm{Cu}$ & 16 & $3 d 1$ & 0.002436 & $-9.8 \mathrm{E}-05$ & -0.00119 & -0.00142 & -0.00146 & 0.003726 \\
\hline $\mathrm{Cu}$ & 16 & $3 d 2$ & -0.04912 & -0.06978 & 0.017635 & 0.094474 & 0.07145 & 0.076605 \\
\hline $\mathrm{Cu}$ & 16 & $3 d 2$ & -0.00326 & -0.00425 & 0.004263 & 0.008531 & 0.003533 & 0.00498 \\
\hline
\end{tabular}




\begin{tabular}{|c|c|c|c|c|c|c|c|c|}
\hline $\mathrm{Cu}$ & 17 & $3 s 0$ & 0.003683 & 0.003975 & 0.000015 & $-2.3 \mathrm{E}-05$ & -0.00157 & 0.000567 \\
\hline $\mathrm{Cu}$ & 17 & $4 s 0$ & -0.08614 & 0.035285 & -0.0002 & 0.000424 & -0.00317 & 0.033308 \\
\hline $\mathrm{Cu}$ & 17 & $4 s 0$ & -0.00115 & -0.00639 & $-3.2 \mathrm{E}-05$ & 0.000022 & 0.00916 & -0.00212 \\
\hline $\mathrm{Cu}$ & 17 & $3 p-1$ & -0.00681 & -0.00204 & -0.00159 & 0.004311 & 0.001357 & 0.008676 \\
\hline $\mathrm{Cu}$ & 17 & $4 p-1$ & 0.030067 & 0.009017 & 0.005168 & -0.02041 & -0.0051 & -0.01661 \\
\hline $\mathrm{Cu}$ & 17 & $3 p 0$ & -0.00686 & 0.007058 & 0.0001 & 0.005843 & 0.002234 & 0.000695 \\
\hline $\mathrm{Cu}$ & 17 & $4 p 0$ & 0.031006 & -0.03469 & -0.00038 & -0.03358 & 0.001946 & -0.01007 \\
\hline $\mathrm{Cu}$ & 17 & $3 p 1$ & 0.00903 & 0.007762 & -0.00059 & -0.00676 & -0.01351 & 0.005092 \\
\hline $\mathrm{Cu}$ & 17 & $4 p 1$ & -0.03565 & -0.03538 & 0.001976 & 0.039971 & 0.055348 & -0.01733 \\
\hline $\mathrm{Cu}$ & 17 & $3 d-2$ & -0.05972 & -0.01838 & -0.10783 & -0.05215 & 0.035615 & -0.09965 \\
\hline $\mathrm{Cu}$ & 17 & $3 d-2$ & -0.00205 & -0.00148 & -0.01485 & -0.0054 & -0.00192 & -0.00888 \\
\hline $\mathrm{Cu}$ & 17 & $3 d-1$ & 0.016453 & -0.02931 & -0.0473 & 0.024978 & -0.02939 & 0.104463 \\
\hline $\mathrm{Cu}$ & 17 & $3 d-1$ & 0.000875 & -0.00262 & -0.00307 & 0.001522 & -0.00132 & 0.01056 \\
\hline $\mathrm{Cu}$ & 17 & $3 d 0$ & -0.07784 & 0.060011 & 0.008124 & 0.055548 & 0.097909 & 0.004543 \\
\hline $\mathrm{Cu}$ & 17 & $3 d 0$ & -0.00434 & 0.005542 & 0.000953 & 0.002512 & 0.016285 & 0.001087 \\
\hline $\mathrm{Cu}$ & 17 & $3 d 1$ & 0.047444 & 0.082735 & -0.01789 & -0.05899 & -0.04 & 0.02759 \\
\hline $\mathrm{Cu}$ & 17 & $3 d 1$ & 0.00103 & 0.008183 & -0.00143 & -0.00468 & -0.01047 & 0.004611 \\
\hline $\mathrm{Cu}$ & 17 & $3 d 2$ & -0.10016 & 0.050337 & -0.07327 & 0.071548 & -0.0863 & -0.04585 \\
\hline $\mathrm{Cu}$ & 17 & $3 d 2$ & -0.00695 & 0.003454 & -0.00983 & 0.007719 & -0.00732 & -0.00311 \\
\hline $\mathrm{Cu}$ & 18 & $3 s 0$ & 0.005444 & 0.004368 & 0.004593 & $-6 \mathrm{E}-06$ & -0.00092 & -0.00125 \\
\hline $\mathrm{Cu}$ & 18 & $4 s 0$ & -0.13224 & 0.02294 & -0.03389 & -0.00019 & -0.0012 & -0.08141 \\
\hline $\mathrm{Cu}$ & 18 & $4 s 0$ & -0.00154 & -0.00642 & -0.00884 & 0.000044 & 0.00549 & 0.004809 \\
\hline $\mathrm{Cu}$ & 18 & $3 p-1$ & & & & & & \\
\hline $\mathrm{Cu}$ & 18 & $4 p-1$ & -0.02421 & -0.02555 & -0.01612 & 0.006611 & 0.036884 & 0.00103 \\
\hline $\mathrm{Cu}$ & 18 & $3 p 0$ & 0.000318 & 0.008111 & 0.008254 & -0.00869 & 0.006648 & -0.0091 \\
\hline $\mathrm{Cu}$ & 18 & $4 p 0$ & 0.000469 & -0.03923 & -0.0367 & & -0.01445 & 0.039945 \\
\hline $\mathrm{Cu}$ & 18 & $3 p 1$ & -0.0067 & 0.003525 & 0.002913 & 0.025149 & -0.01159 & -0.00021 \\
\hline $\mathrm{Cu}$ & 18 & $4 p 1$ & 0.031763 & -0.01663 & -0.01363 & -0.09943 & 0.038179 & 0.009537 \\
\hline $\mathrm{Cu}$ & 18 & $3 d-2$ & 0.006165 & 0.072244 & 0.0279 & -0.04463 & -0.0864 & 0.119066 \\
\hline $\mathrm{Cu}$ & 18 & $3 d-2$ & -0.00154 & 0.00464 & 0.00573 & -0.00618 & -0.0037 & 0.010234 \\
\hline $\mathrm{Cu}$ & 18 & $3 d-1$ & -0.07239 & 0.059509 & -0.0149 & 0.00741 & -0.01605 & -0.03538 \\
\hline $\mathrm{Cu}$ & 18 & $3 d-1$ & -0.0062 & 0.006002 & -0.00282 & 0.003537 & -0.00653 & -0.00591 \\
\hline $\mathrm{Cu}$ & 18 & $3 d 0$ & 0.034859 & 0.077017 & 0.120183 & -0.04004 & 0.102231 & 0.022653 \\
\hline $\mathrm{Cu}$ & 18 & $3 d 0$ & 0.001027 & 0.006262 & 0.014383 & -0.00136 & 0.015073 & -0.0013 \\
\hline $\mathrm{Cu}$ & 18 & $3 d 1$ & -0.02246 & 0.031438 & 0.005059 & 0.055382 & -0.02929 & -0.06803 \\
\hline $\mathrm{Cu}$ & 18 & $3 d 1$ & -0.0021 & 0.004026 & -0.00108 & 0.000553 & -0.00493 & -0.00863 \\
\hline $\mathrm{Cu}$ & 18 & $3 d 2$ & -0.12248 & -0.00454 & 0.026322 & 0.007431 & 0.020904 & -0.00846 \\
\hline $\mathrm{Cu}$ & 18 & $3 d 2$ & -0.00588 & -0.00061 & 0.0029 & 0.002577 & 0.00001 & -0.00094 \\
\hline $\mathrm{Cu}$ & 19 & $3 s 0$ & -0.00202 & 0.004904 & 0.002505 & $-5.2 \mathrm{E}-05$ & -0.00143 & -0.00058 \\
\hline $\mathrm{Cu}$ & 19 & $4 s 0$ & 0.058373 & 0.009616 & -0.01792 & 0.000284 & -0.00285 & -0.03653 \\
\hline $\mathrm{Cu}$ & 19 & $4 s 0$ & 0.000219 & -0.00656 & -0.00483 & 0.000084 & 0.008335 & 0.002247 \\
\hline $\mathrm{Cu}$ & 19 & $3 p-1$ & 0.000573 & 0.008173 & 0.000943 & -0.01807 & -0.00998 & 0.007696 \\
\hline $\mathrm{Cu}$ & 19 & $4 p-1$ & -0.0002 & -0.03576 & -0.00621 & 0.073037 & 0.040848 & -0.0111 \\
\hline $\mathrm{Cu}$ & 19 & $3 p 0$ & 0.001093 & 0.006778 & 0.004499 & -0.0009 & 0.004405 & -0.00542 \\
\hline
\end{tabular}




\begin{tabular}{|c|c|c|c|c|c|c|c|c|}
\hline $\mathrm{Cu}$ & 19 & $4 p 0$ & -0.00568 & -0.03322 & -0.01997 & 0.001008 & -0.0038 & 0.020933 \\
\hline $\mathrm{Cu}$ & 19 & $3 p 1$ & 0.002521 & -0.0034 & -0.00264 & -0.02615 & 0.011841 & 0.004489 \\
\hline $\mathrm{Cu}$ & 19 & $4 p 1$ & -0.0038 & 0.015409 & 0.011114 & 0.099813 & -0.0392 & -0.01403 \\
\hline $\mathrm{Cu}$ & 19 & $3 d-2$ & -0.04518 & -0.0529 & -0.04028 & -0.02873 & 0.082239 & -0.09921 \\
\hline $\mathrm{Cu}$ & 19 & $3 d-2$ & -0.00346 & -0.0033 & -0.00662 & -0.00234 & 0.003077 & -0.00878 \\
\hline $\mathrm{Cu}$ & 19 & $3 d-1$ & 0.080848 & 0.058172 & -0.02922 & -0.03316 & -0.02827 & 0.018668 \\
\hline $\mathrm{Cu}$ & 19 & $3 d-1$ & 0.00351 & 0.005712 & -0.00295 & 0.001677 & -0.00801 & 0.001255 \\
\hline $\mathrm{Cu}$ & 19 & $3 d 0$ & -0.00861 & 0.063225 & 0.068767 & 0.008994 & 0.094754 & 0.015223 \\
\hline $\mathrm{Cu}$ & 19 & $3 d 0$ & $-4.6 \mathrm{E}-05$ & 0.005508 & 0.008261 & 0.000504 & 0.014029 & -0.00042 \\
\hline $\mathrm{Cu}$ & 19 & $3 d 1$ & 0.047267 & -0.04577 & -0.02953 & -0.0208 & 0.01761 & 0.097958 \\
\hline $\mathrm{Cu}$ & 19 & $3 d 1$ & -0.00068 & -0.00465 & -0.00109 & 0.003853 & 0.003485 & 0.010863 \\
\hline $\mathrm{Cu}$ & 19 & $3 d 2$ & 0.213539 & -0.03499 & 0.102589 & 0.013427 & 0.021699 & 0.060357 \\
\hline $\mathrm{Cu}$ & 19 & $3 d 2$ & 0.010977 & -0.00207 & 0.013664 & 0.002199 & 0.002242 & 0.004807 \\
\hline $\mathrm{Cu}$ & 20 & $3 s 0$ & -0.00551 & 0.005332 & -0.00336 & $-4.7 \mathrm{E}-05$ & -0.00228 & 0.001678 \\
\hline $\mathrm{Cu}$ & 20 & $4 s 0$ & 0.147131 & -0.00055 & 0.024012 & 0.000459 & -0.00503 & 0.105461 \\
\hline $\mathrm{Cu}$ & 20 & $4 s 0$ & 0.001054 & -0.00668 & 0.006466 & 0.000127 & 0.013429 & -0.0064 \\
\hline $\mathrm{Cu}$ & 20 & $3 p-1$ & -0.00537 & -0.00428 & -0.00031 & 0.028261 & 0.002074 & 0.007313 \\
\hline $\mathrm{Cu}$ & 20 & $4 p-1$ & 0.021437 & 0.017972 & -0.00062 & -0.10573 & -0.00999 & -0.00938 \\
\hline $\mathrm{Cu}$ & 20 & $3 p 0$ & 0.003258 & 0.006534 & -0.00588 & 0.005415 & -0.00124 & 0.006755 \\
\hline $\mathrm{Cu}$ & 20 & $4 p 0$ & -0.016 & -0.03 & 0.026 & & 0.017832 & \\
\hline $\mathrm{Cu}$ & 20 & $3 p 1$ & 0.006713 & -0.0081 & 0.003593 & -0.00168 & 0.01363 & -0.00359 \\
\hline $\mathrm{Cu}$ & 20 & $4 p 1$ & -0.02208 & 0.035586 & -0.01691 & -0.00027 & -0.06012 & 0.0269 \\
\hline $\mathrm{Cu}$ & 20 & $3 d-2$ & -0.05171 & 0.034865 & 0.073593 & 0.029785 & -0.04081 & 0.043859 \\
\hline $\mathrm{Cu}$ & 20 & $3 d-2$ & -0.00105 & 0.002053 & 0.009304 & 0.002515 & 0.00043 & 0.004362 \\
\hline $\mathrm{Cu}$ & 20 & $3 d-1$ & -0.0505 & -0.02591 & -0.03829 & 0.061001 & -0.01944 & 0.071849 \\
\hline $\mathrm{Cu}$ & 20 & $3 d-1$ & -0.00169 & -0.00206 & -0.00297 & -0.00055 & 0.000027 & 0.006236 \\
\hline $\mathrm{Cu}$ & 20 & $3 d 0$ & -0.00342 & 0.062275 & -0.07472 & 0.024251 & 0.086841 & -0.00547 \\
\hline $\mathrm{Cu}$ & 20 & $3 d 0$ & 0.000707 & 0.005437 & -0.00899 & 0.000854 & 0.015055 & 0.002687 \\
\hline $\mathrm{Cu}$ & 20 & $3 d 1$ & -0.05148 & -0.06631 & 0.011918 & 0.004351 & 0.048235 & -0.06045 \\
\hline $\mathrm{Cu}$ & 20 & $3 d 1$ & -0.00623 & -0.00669 & -0.00036 & 0.002369 & 0.010778 & -0.01068 \\
\hline $\mathrm{Cu}$ & 20 & $3 d 2$ & 0.002716 & 0.04899 & -0.07162 & 0.009328 & -0.0798 & -0.08587 \\
\hline $\mathrm{Cu}$ & 20 & $3 d 2$ & 0.002255 & 0.002883 & -0.01076 & 0.003266 & -0.00711 & -0.00621 \\
\hline $\mathrm{Si}$ & 21 & $1 s 0$ & 0.001259 & 0.000622 & -0.00098 & 0.000000 & 0.000379 & -0.00215 \\
\hline $\mathrm{Si}$ & 21 & $2 s 0$ & 0.011664 & 0.006351 & -0.00934 & 0.000005 & 0.003256 & -0.01957 \\
\hline $\mathrm{Si}$ & 21 & $3 s 0$ & 0.132649 & 0.104947 & -0.11676 & 0.000044 & 0.019849 & -0.14968 \\
\hline $\mathrm{Si}$ & 21 & $3 s 0$ & -0.01608 & -0.01208 & 0.012557 & 0.000004 & -0.00259 & 0.022721 \\
\hline $\mathrm{Si}$ & 21 & $2 p-1$ & 0.002353 & -0.00035 & -0.00134 & 0.003700 & -0.00028 & -0.0014 \\
\hline $\mathrm{Si}$ & 21 & $3 p-1$ & -0.02503 & 0.030736 & -0.04943 & -0.12337 & 0.073325 & 0.168554 \\
\hline $\mathrm{Si}$ & 21 & $3 p-1$ & -0.00824 & -0.00115 & 0.012276 & -0.00691 & -0.00217 & -0.00777 \\
\hline $\mathrm{Si}$ & 21 & $2 p 0$ & 0.00427 & -0.00384 & 0.002499 & 0.003586 & -0.00083 & 0.001443 \\
\hline $\mathrm{Si}$ & 21 & $3 p 0$ & -0.04729 & 0.090366 & -0.07121 & -0.11878 & 0.041172 & -0.06419 \\
\hline $\mathrm{Si}$ & 21 & $3 p 0$ & -0.01482 & 0.009394 & -0.00806 & -0.00676 & 0.007552 & -0.00164 \\
\hline $\mathrm{Si}$ & 21 & $2 p 1$ & -0.00556 & -0.00333 & 0.003448 & -0.00317 & -0.00076 & 0.001192 \\
\hline $\mathrm{Si}$ & 21 & $3 p 1$ & 0.088523 & 0.12019 & -0.13594 & 0.105602 & 0.220451 & 0.015626 \\
\hline
\end{tabular}




\begin{tabular}{|c|c|c|c|c|c|c|c|c|}
\hline $\mathrm{Si}$ & 21 & $3 p 1$ & 0.017675 & 0.004497 & -0.00766 & 0.005922 & -0.00737 & -0.00743 \\
\hline $\mathrm{Si}$ & 21 & $3 d-2$ & 0.0007 & -0.00111 & -0.00616 & 0.004832 & 0.005881 & 0.002906 \\
\hline $\mathrm{Si}$ & 21 & $3 d-1$ & -0.00177 & -0.00054 & -0.00248 & -0.01137 & -0.00486 & 0.000335 \\
\hline $\mathrm{Si}$ & 21 & $3 d 0$ & -0.00502 & -0.00182 & 0.002067 & -0.00057 & -0.00217 & -0.00113 \\
\hline $\mathrm{Si}$ & 21 & $3 d 1$ & 0.007723 & -0.00049 & 0.002152 & 0.005005 & -0.00648 & -0.00963 \\
\hline $\mathrm{Si}$ & 21 & $3 d 2$ & -0.00514 & -0.00219 & 0.00251 & -0.00531 & 0.006092 & -0.0077 \\
\hline $\mathrm{Si}$ & 22 & $1 s 0$ & 0.001255 & 0.000973 & -0.00146 & $-5 \mathrm{E}-06$ & 0.000185 & -0.0037 \\
\hline $\mathrm{Si}$ & 22 & $2 s 0$ & 0.011624 & 0.009596 & -0.01399 & $-4.3 \mathrm{E}-05$ & 0.001592 & -0.03372 \\
\hline $\mathrm{Si}$ & 22 & $3 s 0$ & 0.131997 & 0.141319 & -0.17507 & 0.000022 & 0.009793 & -0.25847 \\
\hline $\mathrm{Si}$ & 22 & $3 s 0$ & -0.01607 & -0.01652 & 0.01882 & 0.000104 & -0.00133 & 0.039201 \\
\hline $\mathrm{Si}$ & 22 & $2 p-1$ & -0.00162 & -0.00378 & 0.004319 & 0.00271 & -0.00038 & 0.001293 \\
\hline $\mathrm{Si}$ & 22 & $3 p-1$ & 0.037884 & 0.133819 & -0.21851 & -0.08982 & 0.116014 & 0.114472 \\
\hline $\mathrm{Si}$ & 22 & $3 p-1$ & 0.00442 & 0.005401 & -0.00512 & -0.00505 & -0.00436 & -0.01627 \\
\hline $\mathrm{Si}$ & 22 & $2 p 0$ & -0.00135 & -0.00404 & 0.003619 & -0.00545 & -0.00045 & 0.002127 \\
\hline $\mathrm{Si}$ & 22 & $3 p 0$ & 0.025727 & 0.095113 & -0.10482 & 0.182317 & 0.009505 & -0.07623 \\
\hline $\mathrm{Si}$ & 22 & $3 p 0$ & 0.00403 & 0.009901 & -0.01153 & 0.010058 & 0.005121 & -0.00386 \\
\hline $\mathrm{Si}$ & 22 & $2 p 1$ & 0.00608 & 0.000061 & -0.0012 & 0.001469 & 0.000034 & -0.00069 \\
\hline $\mathrm{Si}$ & 22 & $3 p 1$ & -0.07937 & -0.00187 & 0.002701 & -0.0494 & 0.004455 & 0.058504 \\
\hline $\mathrm{Si}$ & 22 & $3 p 1$ & -0.02035 & $-8.5 \mathrm{E}-$ & 0.00664 & -0.00269 & -0.00083 & -0.00166 \\
\hline $\mathrm{Si}$ & 22 & $3 d-2$ & -0.0034 & 0.000995 & -0.00286 & 0.004422 & 0.000964 & 0.002924 \\
\hline $\mathrm{Si}$ & 22 & $3 d-1$ & 0.000697 & -0.0002 & 0.001667 & -0.00674 & -0.00724 & -0.01424 \\
\hline $\mathrm{Si}$ & 22 & $3 d 0$ & 0.001829 & -0.00162 & 0.002994 & 0.00994 & 0.002405 & -0.00098 \\
\hline $\mathrm{Si}$ & 22 & $3 d 1$ & 0.011252 & -0.00195 & -0.00144 & -0.00884 & -0.00038 & 0.001527 \\
\hline $\mathrm{Si}$ & 22 & $3 d 2$ & -0.00438 & 0.001419 & 0.002582 & -0.00959 & -0.0086 & 0.00825 \\
\hline $\mathrm{Si}$ & 23 & $1 s 0$ & 0.00009 & 0.000153 & 0.000064 & 0.000009 & -0.00083 & 0.001061 \\
\hline $\mathrm{Si}$ & 23 & $2 s 0$ & 0.000863 & 0.002014 & 0.000617 & 0.000086 & -0.00715 & 0.00968 \\
\hline $\mathrm{Si}$ & 23 & $3 s 0$ & 0.011269 & 0.055713 & 0.007712 & 0.001145 & -0.04391 & 0.07415 \\
\hline $\mathrm{Si}$ & 23 & $3 s 0$ & -0.00136 & -0.0062 & -0.00086 & -0.00013 & 0.005717 & -0.01128 \\
\hline $\mathrm{Si}$ & 23 & $2 p-1$ & 0.002077 & -0.00025 & -0.00282 & -0.00173 & 0.000177 & -0.00204 \\
\hline $\mathrm{Si}$ & 23 & $3 p-1$ & -0.02728 & 0.003959 & -0.0053 & 0.057575 & 0.024731 & 0.182873 \\
\hline $\mathrm{Si}$ & 23 & $3 p-1$ & -0.00692 & 0.000798 & 0.016947 & 0.003269 & -0.00428 & -0.0058 \\
\hline $\mathrm{Si}$ & 23 & $2 p 0$ & -0.00033 & -0.00391 & -0.00022 & $-1.8 \mathrm{E}-05$ & 0.002318 & -0.00059 \\
\hline $\mathrm{Si}$ & 23 & $3 p 0$ & 0.009176 & 0.135426 & 0.010504 & 0.001139 & -0.47747 & -0.02226 \\
\hline $\mathrm{Si}$ & 23 & $3 p 0$ & 0.000705 & 0.00558 & 0.000324 & 0.000037 & 0.007415 & 0.00481 \\
\hline $\mathrm{Si}$ & 23 & $2 p 1$ & 0.008045 & -0.00102 & -0.00104 & -0.00078 & 0.000051 & -0.00064 \\
\hline $\mathrm{Si}$ & 23 & $3 p 1$ & -0.10489 & 0.013287 & -0.00191 & 0.025777 & 0.01103 & 0.057443 \\
\hline $\mathrm{Si}$ & 23 & $3 p 1$ & -0.02697 & 0.003417 & 0.00622 & 0.001502 & -0.00163 & -0.00183 \\
\hline $\mathrm{Si}$ & 23 & $3 d-2$ & 0.005156 & 0.000951 & $-9.9 \mathrm{E}-05$ & 0.011657 & -0.00015 & 0.000038 \\
\hline $\mathrm{Si}$ & 23 & $3 d-1$ & 0.00069 & -0.00012 & -0.00853 & -0.00046 & 0.004026 & 0.009313 \\
\hline $\mathrm{Si}$ & 23 & $3 d 0$ & 0.000065 & -0.00304 & 0.000172 & 0.000035 & -0.00222 & 0.004303 \\
\hline $\mathrm{Si}$ & 23 & $3 d 1$ & 0.002784 & -0.00037 & -0.00311 & -0.00028 & 0.001549 & 0.002902 \\
\hline $\mathrm{Si}$ & 23 & $3 d 2$ & -0.00856 & -0.0015 & $-1.1 \mathrm{E}-05$ & -0.02371 & -0.00251 & 0.000092 \\
\hline $\mathrm{Si}$ & 24 & $1 s 0$ & -0.00043 & 0.001158 & -0.00156 & $-4 \mathrm{E}-06$ & $-5.7 \mathrm{E}-05$ & -0.00471 \\
\hline $\mathrm{Si}$ & 24 & $2 s 0$ & -0.00394 & 0.011304 & -0.01497 & $-3.8 \mathrm{E}-05$ & -0.0005 & -0.04292 \\
\hline
\end{tabular}




\begin{tabular}{|c|c|c|c|c|c|c|c|c|}
\hline $\mathrm{Si}$ & 24 & $3 s 0$ & -0.04241 & 0.160645 & -0.18751 & -0.00057 & -0.00318 & -0.32856 \\
\hline $\mathrm{Si}$ & 24 & $3 s 0$ & 0.00521 & -0.01885 & 0.020105 & 0.000023 & 0.000449 & 0.049877 \\
\hline $\mathrm{Si}$ & 24 & $2 p-1$ & 0.005361 & -0.00338 & 0.003344 & -0.00393 & 0.000195 & 0.001411 \\
\hline $\mathrm{Si}$ & 24 & $3 p-1$ & -0.07058 & 0.114458 & -0.18968 & 0.131724 & -0.00021 & 0.108076 \\
\hline $\mathrm{Si}$ & 24 & $3 p-1$ & -0.01805 & 0.005338 & -0.00214 & 0.007234 & -0.00242 & -0.01648 \\
\hline $\mathrm{Si}$ & 24 & $2 p 0$ & 0.004418 & 0.00367 & -0.00371 & -0.00567 & 0.000391 & -0.00166 \\
\hline $\mathrm{Si}$ & 24 & $3 p 0$ & -0.05708 & -0.09198 & 0.115254 & 0.189726 & 0.084959 & 0.003016 \\
\hline $\mathrm{Si}$ & 24 & $3 p 0$ & -0.0148 & -0.00856 & 0.011116 & 0.010445 & -0.01168 & 0.008024 \\
\hline $\mathrm{Si}$ & 24 & $2 p 1$ & -0.00275 & -0.00261 & 0.00342 & 0.000592 & 0.000308 & 0.001811 \\
\hline $\mathrm{Si}$ & 24 & $3 p 1$ & 0.035359 & 0.084019 & -0.13504 & -0.02052 & 0.029278 & 0.003755 \\
\hline $\mathrm{Si}$ & 24 & $3 p 1$ & 0.009183 & 0.004509 & -0.00751 & -0.00106 & -0.00626 & -0.00942 \\
\hline $\mathrm{Si}$ & 24 & $3 d-2$ & 0.005859 & -0.00183 & -0.00157 & -0.00079 & 0.009107 & -0.01284 \\
\hline $\mathrm{Si}$ & 24 & $3 d-1$ & 0.001102 & 0.000318 & -0.00047 & -0.00606 & 0.009138 & 0.011387 \\
\hline $\mathrm{Si}$ & 24 & $3 d 0$ & 0.005762 & -0.00213 & 0.002703 & -0.00159 & -0.00412 & 0.002146 \\
\hline $\mathrm{Si}$ & 24 & $3 d 1$ & 0.008709 & -0.00152 & -0.00159 & 0.005278 & 0.004105 & 0.009873 \\
\hline $\mathrm{Si}$ & 24 & $3 d 2$ & -0.00075 & -0.00018 & 0.002842 & 0.004982 & $-6.1 \mathrm{E}-05$ & 0.00176 \\
\hline $\mathrm{Si}$ & 25 & $1 s 0$ & 0.000394 & 0.00075 & 0.000902 & $-4 \mathrm{E}-06$ & 0.00001 & 0.003093 \\
\hline $\mathrm{Si}$ & 25 & $2 s 0$ & 0.003673 & 0.007528 & 0.008636 & $-3.4 \mathrm{E}-05$ & 0.000076 & 0.028204 \\
\hline $\mathrm{Si}$ & 25 & $3 s 0$ & 0.042786 & 0.117997 & 0.108071 & -0.00027 & 0.000344 & 0.215767 \\
\hline $\mathrm{Si}$ & 25 & $3 s 0$ & -0.00519 & -0.01371 & -0.0116 & 0.000065 & $-1.5 \mathrm{E}-05$ & -0.03276 \\
\hline $\mathrm{Si}$ & 25 & $2 p-1$ & 0.00083 & 0.002166 & 0.00062 & -0.00079 & $-1.1 \mathrm{E}-05$ & 0.00019 \\
\hline $\mathrm{Si}$ & 25 & $3 p-1$ & & -0.09386 & -0.10826 & 0.026532 & -0.01742 & 0.132386 \\
\hline $\mathrm{Si}$ & 25 & $3 p-1$ & -0.00236 & -0.00157 & 0.006258 & 0.001472 & 0.001489 & -0.01264 \\
\hline $\mathrm{Si}$ & 25 & $2 p 0$ & 0.001883 & -0.00365 & -0.00211 & 0.000815 & -0.00042 & -0.00093 \\
\hline $\mathrm{Si}$ & 25 & $3 p 0$ & -0.02071 & 0.088971 & 0.067062 & -0.02695 & -0.06203 & -0.01106 \\
\hline $\mathrm{Si}$ & 25 & $3 p 0$ & -0.00659 & 0.008657 & 0.006149 & -0.00157 & 0.010326 & 0.005668 \\
\hline $\mathrm{Si}$ & 25 & $2 p 1$ & -0.00197 & -0.00306 & -0.00371 & -0.00173 & 0.000239 & -0.00231 \\
\hline $\mathrm{Si}$ & 25 & $3 p 1$ & 0.030631 & 0.089168 & 0.078435 & 0.057915 & 0.052264 & 0.094586 \\
\hline $\mathrm{Si}$ & 25 & $3 p 1$ & 0.006217 & 0.00605 & 0.014406 & 0.003197 & -0.00715 & 0.003363 \\
\hline $\mathrm{Si}$ & 25 & $3 d-2$ & 0.004256 & 0.00236 & 0.000601 & 0.004773 & -0.00951 & -0.01033 \\
\hline $\mathrm{Si}$ & 25 & $3 d-1$ & 0.007191 & -0.00048 & -0.00143 & 0.006508 & 0.007984 & -0.00528 \\
\hline $\mathrm{Si}$ & 25 & $3 d 0$ & -0.00218 & -0.0021 & -0.00138 & -0.00309 & -0.00286 & -0.00205 \\
\hline $\mathrm{Si}$ & 25 & $3 d 1$ & 0.013986 & -0.00093 & -0.00303 & 0.011981 & -0.00482 & 0.008534 \\
\hline $\mathrm{Si}$ & 25 & $3 d 2$ & -0.00733 & 0.000774 & -0.00699 & -0.00607 & 0.002062 & 0.004259 \\
\hline $\mathrm{Si}$ & 26 & $1 s 0$ & -0.00235 & 0.000879 & -0.00011 & $-1.4 \mathrm{E}-05$ & 0.000253 & -0.00048 \\
\hline $\mathrm{Si}$ & 26 & $2 s 0$ & -0.02172 & 0.008726 & -0.00102 & -0.00014 & 0.002173 & -0.00434 \\
\hline $\mathrm{Si}$ & 26 & $3 s 0$ & -0.24164 & 0.131421 & -0.01277 & -0.00156 & 0.013299 & -0.03341 \\
\hline $\mathrm{Si}$ & 26 & $3 s 0$ & 0.029558 & -0.01533 & 0.00136 & 0.000129 & -0.00173 & 0.004994 \\
\hline $\mathrm{Si}$ & 26 & $2 p-1$ & -0.00329 & 0.001477 & -0.00308 & 0.000047 & 0.000144 & -0.00217 \\
\hline $\mathrm{Si}$ & 26 & $3 p-1$ & 0.048743 & -0.04446 & 0.001994 & -0.00131 & -0.05124 & 0.185677 \\
\hline $\mathrm{Si}$ & 26 & $3 p-1$ & 0.010832 & -0.00277 & 0.017637 & $-9.9 \mathrm{E}-05$ & 0.002197 & -0.00541 \\
\hline $\mathrm{Si}$ & 26 & $2 p 0$ & 0.000285 & 0.002787 & -0.00013 & 0.006749 & 0.000752 & 0.000269 \\
\hline $\mathrm{Si}$ & 26 & $3 p 0$ & 0.006691 & -0.07783 & 0.007792 & -0.22426 & 0.001224 & -0.03804 \\
\hline $\mathrm{Si}$ & 26 & $3 p 0$ & -0.00143 & -0.00577 & 0.000039 & -0.01258 & -0.00973 & 0.001963 \\
\hline
\end{tabular}




\begin{tabular}{|c|c|c|c|c|c|c|c|c|}
\hline $\mathrm{Si}$ & 26 & $2 p 1$ & 0.003166 & -0.00398 & -0.00056 & 0.003591 & -0.00043 & -0.00023 \\
\hline $\mathrm{Si}$ & 26 & $3 p 1$ & -0.061 & 0.131871 & -0.01605 & -0.12046 & 0.16395 & 0.049177 \\
\hline $\mathrm{Si}$ & 26 & $3 p 1$ & -0.00974 & 0.006439 & 0.004722 & -0.00666 & -0.00722 & -0.00309 \\
\hline $\mathrm{Si}$ & 26 & $3 d-2$ & 0.004536 & 0.001666 & -0.00667 & -0.00195 & -0.00602 & 0.0085 \\
\hline $\mathrm{Si}$ & 26 & $3 d-1$ & 0.005092 & -0.00055 & 0.00392 & 0.003747 & -0.00546 & -0.00407 \\
\hline $\mathrm{Si}$ & 26 & $3 d 0$ & 0.000533 & -0.00316 & -0.00011 & 0.007618 & -0.00386 & 0.001601 \\
\hline $\mathrm{Si}$ & 26 & $3 d 1$ & 0.006492 & -0.00121 & 0.001384 & 0.007139 & 0.007341 & -0.00111 \\
\hline $\mathrm{Si}$ & 26 & $3 d 2$ & -0.00206 & -0.00303 & -0.00465 & 0.008192 & 0.006129 & 0.003146 \\
\hline $\mathrm{Si}$ & 27 & $1 s 0$ & -0.00043 & 0.001158 & 0.001563 & 0.000002 & -0.00006 & 0.004707 \\
\hline $\mathrm{Si}$ & 27 & $2 s 0$ & -0.00395 & 0.011301 & 0.014963 & 0.000024 & -0.00052 & 0.042921 \\
\hline $\mathrm{Si}$ & 27 & $3 s 0$ & -0.0425 & 0.160629 & 0.187483 & 0.000497 & -0.00339 & 0.328593 \\
\hline $\mathrm{Si}$ & 27 & $3 s 0$ & 0.005218 & -0.01884 & -0.0201 & -2E-06 & 0.000475 & -0.04989 \\
\hline $\mathrm{Si}$ & 27 & $2 p-1$ & -0.00537 & 0.003373 & 0.003344 & -0.00393 & -0.00019 & 0.001411 \\
\hline $\mathrm{Si}$ & 27 & $3 p-1$ & 0.070629 & -0.1144 & -0.18964 & 0.131587 & 0.000174 & 0.107929 \\
\hline $\mathrm{Si}$ & 27 & $3 p-1$ & 0.018074 & -0.00533 & -0.00214 & 0.007236 & 0.00243 & -0.01646 \\
\hline $\mathrm{Si}$ & 27 & $2 p 0$ & -0.00442 & -0.00367 & -0.00372 & -0.00566 & -0.00038 & -0.00167 \\
\hline $\mathrm{Si}$ & 27 & $3 p 0$ & 0.056972 & 0.092001 & 0.115283 & 0.189597 & -0.08555 & 0.002962 \\
\hline $\mathrm{Si}$ & 27 & $3 p 0$ & 0.01481 & 0.008566 & 0.011127 & 0.010432 & 0.011695 & 0.008057 \\
\hline $\mathrm{Si}$ & 27 & $2 p 1$ & 0.002754 & 0.002614 & 0.003415 & 0.000592 & -0.00031 & 0.001808 \\
\hline $\mathrm{Si}$ & 27 & $3 p 1$ & -0.03531 & -0.0841 & -0.135 & -0.02048 & -0.0292 & 0.004093 \\
\hline $\mathrm{Si}$ & 27 & $3 p 1$ & -0.00919 & -0.00451 & -0.00749 & -0.00106 & 0.006266 & -0.00941 \\
\hline $\mathrm{Si}$ & 27 & $3 d-2$ & & -0.00182 & 0.001583 & 0.000819 & & 0.012844 \\
\hline $\mathrm{Si}$ & 27 & $3 d-1$ & 0.001083 & 0.000311 & 0.000485 & 0.006066 & 0.009149 & -0.01137 \\
\hline $\mathrm{Si}$ & 27 & $3 d 0$ & 0.005758 & -0.00213 & -0.00269 & 0.00162 & -0.00417 & -0.00218 \\
\hline $\mathrm{Si}$ & 27 & $3 d 1$ & 0.008731 & -0.00151 & 0.001588 & -0.00526 & 0.004119 & -0.00988 \\
\hline $\mathrm{Si}$ & 27 & $3 d 2$ & -0.00074 & -0.00017 & -0.00285 & -0.00497 & $-6.2 \mathrm{E}-05$ & -0.00175 \\
\hline $\mathrm{Si}$ & 28 & $1 s 0$ & 0.001257 & 0.000974 & 0.001465 & 0.000002 & 0.000171 & 0.003699 \\
\hline $\mathrm{Si}$ & 28 & $2 s 0$ & 0.011645 & 0.009599 & 0.014021 & 0.000011 & 0.001466 & 0.033734 \\
\hline $\mathrm{Si}$ & 28 & $3 s 0$ & 0.132233 & 0.14137 & 0.175312 & -0.00031 & 0.008816 & 0.258563 \\
\hline $\mathrm{Si}$ & 28 & $3 s 0$ & -0.0161 & -0.01652 & -0.01886 & $-5.6 \mathrm{E}-05$ & -0.00118 & -0.03922 \\
\hline $\mathrm{Si}$ & 28 & $2 p-1$ & 0.001627 & 0.003774 & 0.004326 & 0.002707 & 0.000368 & 0.001296 \\
\hline $\mathrm{Si}$ & 28 & $3 p-1$ & -0.03798 & -0.13382 & -0.21859 & -0.08978 & -0.11646 & 0.11453 \\
\hline $\mathrm{Si}$ & 28 & $3 p-1$ & -0.00444 & -0.0054 & -0.00514 & -0.00504 & 0.00442 & -0.01627 \\
\hline $\mathrm{Si}$ & 28 & $2 p 0$ & 0.00134 & 0.004046 & 0.00361 & -0.00545 & 0.000449 & 0.002123 \\
\hline $\mathrm{Si}$ & 28 & $3 p 0$ & -0.02569 & -0.09519 & -0.10471 & 0.18241 & -0.00998 & -0.07582 \\
\hline $\mathrm{Si}$ & 28 & $3 p 0$ & -0.00401 & -0.00991 & -0.0115 & 0.010065 & -0.00509 & -0.00387 \\
\hline $\mathrm{Si}$ & 28 & $2 p 1$ & -0.00607 & $-6.7 \mathrm{E}-05$ & -0.0012 & 0.001464 & $-3.3 \mathrm{E}-05$ & -0.00069 \\
\hline $\mathrm{Si}$ & 28 & $3 p 1$ & 0.079219 & 0.001996 & 0.002687 & -0.04934 & -0.00453 & 0.058141 \\
\hline $\mathrm{Si}$ & 28 & $3 p 1$ & 0.020336 & 0.000103 & 0.00664 & -0.00268 & 0.000825 & -0.00163 \\
\hline $\mathrm{Si}$ & 28 & $3 d-2$ & -0.00341 & 0.000991 & 0.002868 & -0.00441 & 0.00096 & -0.0029 \\
\hline $\mathrm{Si}$ & 28 & $3 d-1$ & 0.000704 & -0.00019 & -0.00166 & 0.006736 & -0.00728 & 0.014233 \\
\hline $\mathrm{Si}$ & 28 & $3 d 0$ & 0.001835 & -0.00163 & -0.003 & -0.00993 & 0.002435 & 0.000984 \\
\hline $\mathrm{Si}$ & 28 & $3 d 1$ & 0.011256 & -0.00194 & 0.001427 & 0.00883 & -0.00038 & -0.00152 \\
\hline $\mathrm{Si}$ & 28 & $3 d 2$ & -0.00438 & 0.001422 & -0.00259 & 0.009603 & -0.00857 & -0.00826 \\
\hline
\end{tabular}




\begin{tabular}{|c|c|c|c|c|c|c|c|c|}
\hline $\mathrm{Si}$ & 29 & $1 s 0$ & -0.00236 & 0.000879 & 0.000107 & 0.000009 & 0.000257 & 0.000475 \\
\hline $\mathrm{Si}$ & 29 & $2 s 0$ & -0.02173 & 0.008724 & 0.001026 & 0.000088 & 0.002215 & 0.004335 \\
\hline $\mathrm{Si}$ & 29 & $3 s 0$ & -0.24183 & 0.131391 & 0.012798 & 0.001151 & 0.013669 & 0.033384 \\
\hline $\mathrm{Si}$ & 29 & $3 s 0$ & 0.02958 & -0.01533 & -0.00137 & -0.00007 & -0.00178 & -0.00499 \\
\hline $\mathrm{Si}$ & 29 & $2 p-1$ & 0.00329 & -0.00148 & -0.00308 & 0.000044 & -0.00014 & -0.00216 \\
\hline $\mathrm{Si}$ & 29 & $3 p-1$ & -0.04872 & 0.044461 & 0.002038 & -0.00113 & 0.050659 & 0.185431 \\
\hline $\mathrm{Si}$ & 29 & $3 p-1$ & -0.01082 & 0.002788 & 0.017641 & $-9.8 \mathrm{E}-05$ & -0.00218 & -0.0054 \\
\hline $\mathrm{Si}$ & 29 & $2 p 0$ & -0.0003 & -0.00279 & -0.00012 & 0.006749 & -0.00075 & 0.000265 \\
\hline $\mathrm{Si}$ & 29 & $3 p 0$ & -0.00658 & 0.077921 & 0.007714 & -0.22429 & -0.00174 & -0.03854 \\
\hline $\mathrm{Si}$ & 29 & $3 p 0$ & 0.001457 & 0.005778 & 0.000026 & -0.01258 & 0.009745 & 0.002014 \\
\hline $\mathrm{Si}$ & 29 & $2 p 1$ & -0.00318 & 0.003974 & -0.00056 & 0.003589 & 0.000434 & -0.00023 \\
\hline $\mathrm{Si}$ & 29 & $3 p 1$ & 0.061272 & -0.13178 & -0.01606 & -0.12055 & -0.16372 & 0.049585 \\
\hline $\mathrm{Si}$ & 29 & $3 p 1$ & 0.009776 & -0.00643 & 0.004714 & -0.00664 & 0.007205 & -0.00307 \\
\hline $\mathrm{Si}$ & 29 & $3 d-2$ & 0.004538 & 0.001663 & 0.006673 & 0.001958 & -0.00601 & -0.00848 \\
\hline $\mathrm{Si}$ & 29 & $3 d-1$ & 0.005084 & -0.00056 & -0.00391 & -0.00374 & -0.00546 & 0.004067 \\
\hline $\mathrm{Si}$ & 29 & $3 d 0$ & 0.000523 & -0.00315 & 0.000098 & -0.00759 & -0.00391 & -0.00165 \\
\hline $\mathrm{Si}$ & 29 & $3 d 1$ & 0.006493 & -0.00122 & -0.00138 & -0.00712 & 0.007314 & 0.001125 \\
\hline $\mathrm{Si}$ & 29 & $3 d 2$ & -0.00206 & -0.00304 & 0.004649 & -0.00819 & 0.006147 & -0.00314 \\
\hline $\mathrm{Si}$ & 30 & $1 s 0$ & 0.001259 & 0.000623 & 0.000976 & 0.000001 & 0.000372 & 0.002145 \\
\hline $\mathrm{Si}$ & 30 & & 0.011 & & & & & \\
\hline $\mathrm{Si}$ & 30 & $3 s 0$ & 0.132651 & 0.104957 & 0.116696 & 0.000126 & 0.019308 & 0.149578 \\
\hline $\mathrm{Si}$ & 30 & $3 s 0$ & -0.0161 & -0.01208 & -0.01255 & $-2.4 \mathrm{E}-05$ & -0.00252 & -0.02271 \\
\hline $\mathrm{Si}$ & 30 & $2 p-1$ & -0.00235 & 0.000348 & -0.00135 & 0.003698 & 0.000286 & -0.0014 \\
\hline $\mathrm{Si}$ & 30 & $3 p-1$ & 0.025045 & -0.03084 & -0.04931 & -0.12337 & -0.07383 & 0.168208 \\
\hline $\mathrm{Si}$ & 30 & $3 p-1$ & 0.00823 & 0.001143 & 0.012305 & -0.0069 & 0.002195 & -0.00777 \\
\hline $\mathrm{Si}$ & 30 & $2 p 0$ & -0.00427 & 0.003842 & 0.002502 & 0.003583 & 0.00083 & 0.001443 \\
\hline $\mathrm{Si}$ & 30 & $3 p 0$ & 0.047303 & -0.09037 & -0.07125 & -0.11875 & -0.04185 & -0.06472 \\
\hline $\mathrm{Si}$ & 30 & $3 p 0$ & 0.014825 & -0.0094 & -0.00806 & -0.00674 & -0.00751 & -0.00164 \\
\hline $\mathrm{Si}$ & 30 & $2 p 1$ & 0.005563 & 0.003331 & 0.003451 & -0.00316 & 0.000759 & 0.001192 \\
\hline $\mathrm{Si}$ & 30 & $3 p 1$ & -0.08852 & -0.12014 & -0.13594 & 0.105437 & -0.22044 & 0.014358 \\
\hline $\mathrm{Si}$ & 30 & $3 p 1$ & -0.01769 & -0.0045 & -0.00767 & 0.005908 & 0.007381 & -0.00738 \\
\hline $\mathrm{Si}$ & 30 & $3 d-2$ & 0.000689 & -0.00111 & 0.006159 & -0.00481 & 0.005887 & -0.00289 \\
\hline $\mathrm{Si}$ & 30 & $3 d-1$ & -0.00174 & -0.00054 & 0.002486 & 0.011344 & -0.00486 & -0.00033 \\
\hline $\mathrm{Si}$ & 30 & $3 d 0$ & -0.00501 & -0.00183 & -0.00206 & 0.000584 & -0.00213 & 0.001164 \\
\hline $\mathrm{Si}$ & 30 & $3 d 1$ & 0.007719 & -0.00049 & -0.00216 & -0.00501 & -0.0065 & 0.009608 \\
\hline $\mathrm{Si}$ & 30 & $3 d 2$ & -0.00514 & -0.0022 & -0.00251 & 0.005321 & 0.006059 & 0.007729 \\
\hline $\mathrm{Si}$ & 31 & $1 s 0$ & 0.000394 & 0.000749 & -0.00091 & 0.000002 & 0.000013 & -0.0031 \\
\hline $\mathrm{Si}$ & 31 & $2 s 0$ & 0.003674 & 0.007521 & -0.00868 & 0.000021 & 0.000105 & -0.02823 \\
\hline $\mathrm{Si}$ & 31 & $3 s 0$ & 0.042818 & 0.117964 & -0.10841 & 0.000118 & 0.000542 & -0.21599 \\
\hline $\mathrm{Si}$ & 31 & $3 s 0$ & -0.00519 & -0.01369 & 0.01166 & $-4.9 \mathrm{E}-05$ & $-5.5 \mathrm{E}-05$ & 0.032788 \\
\hline $\mathrm{Si}$ & 31 & $2 p-1$ & -0.00083 & -0.00216 & 0.000627 & -0.00079 & 0.000012 & 0.000194 \\
\hline $\mathrm{Si}$ & 31 & $3 p-1$ & 0.016922 & 0.093714 & -0.10838 & 0.026525 & 0.01732 & 0.132103 \\
\hline $\mathrm{Si}$ & 31 & $3 p-1$ & 0.002365 & 0.001531 & 0.006239 & 0.001468 & -0.00147 & -0.01263 \\
\hline $\mathrm{Si}$ & 31 & $2 p 0$ & -0.00187 & 0.003649 & -0.00211 & 0.000813 & 0.000429 & -0.00093 \\
\hline
\end{tabular}




\begin{tabular}{lllllllll}
\hline $\mathrm{Si}$ & 31 & $3 p 0$ & 0.020478 & -0.089 & 0.067142 & -0.02689 & 0.061335 & -0.01123 \\
$\mathrm{Si}$ & 31 & $3 p 0$ & 0.006586 & -0.00865 & 0.006152 & -0.00156 & -0.01031 & 0.005687 \\
$\mathrm{Si}$ & 31 & $2 p 1$ & 0.001965 & 0.003074 & -0.00371 & -0.00173 & -0.00023 & -0.0023 \\
$\mathrm{Si}$ & 31 & $3 p 1$ & -0.03056 & -0.08931 & 0.078341 & 0.057956 & -0.05257 & 0.094102 \\
$\mathrm{Si}$ & 31 & $3 p 1$ & -0.00621 & -0.00608 & 0.014405 & 0.003199 & 0.007137 & 0.003373 \\
$\mathrm{Si}$ & 31 & $3 d-2$ & 0.004265 & 0.002362 & -0.00059 & -0.00477 & -0.00953 & 0.010311 \\
$\mathrm{Si}$ & 31 & $3 d-1$ & 0.007185 & -0.00049 & 0.001445 & -0.00651 & 0.007977 & 0.005283 \\
$\mathrm{Si}$ & 31 & $3 d 0$ & -0.00219 & -0.0021 & 0.001387 & 0.003096 & -0.00282 & 0.002075 \\
$\mathrm{Si}$ & 31 & $3 d 1$ & 0.01399 & -0.00093 & 0.003029 & -0.01198 & -0.0048 & -0.00855 \\
$\mathrm{Si}$ & 31 & $3 d 2$ & -0.00734 & 0.000769 & 0.007002 & 0.006084 & 0.002075 & -0.00424 \\
$\mathrm{Si}$ & 32 & $1 s 0$ & 0.000091 & 0.000155 & $-6.3 \mathrm{E}-05$ & $-6 \mathrm{E}-06$ & -0.00085 & -0.00106 \\
$\mathrm{Si}$ & 32 & $2 s 0$ & 0.00087 & 0.002025 & -0.00061 & $-6.5 \mathrm{E}-05$ & -0.00733 & -0.00966 \\
$\mathrm{Si}$ & 32 & $3 s 0$ & 0.011443 & 0.055785 & -0.0076 & -0.00091 & -0.04528 & -0.07401 \\
$\mathrm{Si}$ & 32 & $3 s 0$ & -0.00137 & -0.00622 & 0.00084 & 0.0001 & 0.005911 & 0.011259 \\
$\mathrm{Si}$ & 32 & $2 p-1$ & -0.00209 & 0.000249 & -0.00282 & -0.00173 & -0.00017 & -0.00203 \\
$\mathrm{Si}$ & 32 & $3 p-1$ & 0.027383 & -0.00387 & -0.00517 & 0.057571 & -0.02516 & 0.182941 \\
$\mathrm{Si}$ & 32 & $3 p-1$ & 0.006947 & -0.00079 & 0.016967 & 0.003266 & 0.004287 & -0.00581 \\
$\mathrm{Si}$ & 32 & $2 p 0$ & 0.000332 & 0.003909 & -0.00022 & $-1.1 \mathrm{E}-05$ & -0.00233 & -0.00059 \\
$\mathrm{Si}$ & 32 & $3 p 0$ & -0.0093 & -0.13541 & 0.010505 & 0.000877 & 0.477926 & -0.02169 \\
$\mathrm{Si}$ & 32 & $3 p 0$ & -0.00071 & -0.00559 & 0.000322 & 0.000027 & -0.00737 & 0.004793 \\
$\mathrm{Si}$ & 32 & $2 p 1$ & -0.00803 & 0.001021 & -0.00104 & -0.00079 & $-5.3 \mathrm{E}-05$ & -0.00064 \\
$\mathrm{Si}$ & 32 & $3 p 1$ & 0.104909 & -0.01325 & -0.00189 & 0.025778 & -0.01094 & 0.057212 \\
$\mathrm{Si}$ & 32 & $3 p 1$ & 0.026925 & -0.00341 & 0.00621 & 0.00154 & 0.00163 & -0.00179 \\
$\mathrm{Si}$ & 32 & $3 d-2$ & 0.005127 & 0.000951 & 0.000108 & -0.01165 & -0.00014 & $-6.2 \mathrm{E}-05$ \\
$\mathrm{Si}$ & 32 & $3 d-1$ & 0.000695 & -0.00012 & 0.008528 & 0.00046 & 0.004044 & -0.00931 \\
$\mathrm{Si}$ & 32 & $3 d 0$ & 0.000067 & -0.00305 & -0.00016 & $-2.5 \mathrm{E}-05$ & -0.0023 & -0.0043 \\
$\mathrm{Si}$ & 32 & $3 d 1$ & 0.002792 & -0.00037 & 0.00311 & 0.000283 & 0.001539 & -0.00286 \\
$\mathrm{Si}$ & 32 & $3 d 2$ & -0.00857 & -0.0015 & 0.000004 & 0.023715 & -0.00250 & -0.00013 \\
\hline & & & & & & & & \\
& & & & & & & \\
& & & &
\end{tabular}

